\title{
A Comprehensive Review of Wearable Applications and Material Construction
}

\author{
Shenhao Wang \\ School of Physics, University of Electronic Science and Technology of China, Chengdu, China \\ Email: 1371546789@qq.com
}

How to cite this paper: Wang, S.H. (2020) A Comprehensive Review of Wearable Applications and Material Construction. Open Journal of Applied Sciences, 10, 364-408. https://doi.org/10.4236/ojapps.2020.106027

Received: March 3, 2020

Accepted: June 27, 2020

Published: June 30, 2020

Copyright $\odot 2020$ by author(s) and Scientific Research Publishing Inc. This work is licensed under the Creative Commons Attribution International License (CC BY 4.0).

http://creativecommons.org/licenses/by/4.0/

\begin{abstract}
Wearable electronic systems are able to monitor and measure multiple biophysical, biochemical signals to help researchers develop further understandings of human health and correlation between human performance and diseases. Driven by increasing demand for need in sports training, health monitoring and disease diagnose, bio-integrated systems are developing at a significant speed based on recent advances in material science, structure design and chemical techniques. A wide range of wearable systems are created and feature unique measuring targets, methods and soft, transparent, stretchable characters. This review summarizes the recent advances in wearable electronic technologies that also include material science, chemical science and electronic engineering. The introduction to basic wearable fundamentals covers subsequent consideration for materials, system integration and promising platforms. Detailed classification towards their functions of physical, chemical detection is also mentioned. Strategies to achieve stretchability and promising material, AgNW, are fully discussed. This paper concludes with consideration of main challenging obstacles in this emerging filed and promises in materials that possess excellent potentials for predicted progress.
\end{abstract}

\section{Keywords}

Wearable Electronics, Stretchable Material, Silver Nanowires

\section{Introduction}

Body systems are running all the time, generating a wide range of signals that reflect every single massage of this bio-system. The biochemical and biophysical signals can be collected and analyzed to develop further insights into personal health and unknown diseases. Through direct contact with human body, wearable electronics are able to develop specific analyze toward target bio-fluids meas- 
ured by sensors. There are already a significant number of such wearable systems in the lab and market. Though the accuracy may suffer a blow when compared with those traditional, sophisticated clinical analytic machines, wearable systems possesses incredible timeliness, personal care and potentials for health monitoring.

Although the challenge of stable, intimate and accurate skin interfaces with stretchable components has not been addressed, this does not influence the high spirit for customers to the wearable fitness and wellness monitoring devices. The application for basic biophysical signal monitor, such as heart rate monitor, temperature monitor and motion monitor, is now prevailing among the electronic devices market. Apple watch features its heart rate monitoring, and can achieve accuracy comparable to clinical monitors for patients, besides it can also detect abnormal beating styles, sending alerts for users. Other wearable systems also cover wide range of fields, e.g., smart plant sensors are introduced by nano-biotechnology approaches to have solid monitoring strategies and maximize the output of crops [1].

Recent advances in material, chemical science and electronic engineering provide a new aspect of promising potential of wearable electronics. Stretchability and planarity are newly developed demands for wearable interfaces with developing engineering technologies and material analyze technologies. In consideration of stretchability, two basic methods (constructing stretchable material and constructing stretchable constructs) are under use at present, and contribute to most reported systems. Carbon Nanomaterials (e.g., Carbon Blacks, Carbon Nanotube), Conductive Polymer (polypyrrole and PEDOT-based materials), Nanowires (NWs) and Nanoparticles (NPs) are the popular materials with latent stretchability for wearable electrodes' designs. Stretchable structures are explained to allow the skin interfaces deform as the users moves (e.g., bending their wrists), which include mesh-shaped or buckled structures, Open-mesh structure, Coiled Structure and Sponge Structure [2].

This review underlines the latest advances of wearable technologies, with main emphasis on materials and structures that lead to potential stretchability of the future interfaces. Other reviews present the filed in the context of chemistry/ physical concepts (signal processing [3], bio-analyte [4]), specific applications (biochemical monitoring [1], workload monitoring [5], smart skins [6], health diagnostics [7], healthcare monitoring [8] [9]), fabrication methods [2], energy resources (energy autonomous skin [10], wearable power Source [11]). By contrast, this review presents the recent advances in a comprehensive way, and connects the wearable technologies with the material fabrications and structure designs with easy accessible examples. The first section contextualizes the vital considerations of platform and sensor designing with introduction to the emerging filed of wearable electronics. In subsequent sections, the construction for stretchable materials and structures are highlighted to create significant understanding for material fabrications. Additional section features the superiority of 
AgNW for sensor building among other promising materials and conclusions toward recent wearable advances are drawn with a summary of latent chances in material science and electronic engineering.

\section{Basic Definition and Mechanics of Wearable Sensors}

Wearable electronics could be defined as wearable devices that utilize computers to complete particular functions. They are usually worn by users, and are known as some popular products, such as apple watches, google glasses. However, literally even clothes, shoes, accessories and those able to be worn have possibility to be developed into wearable electronics. The essential key of every wearable electronics, regardless of what specific application it developed for, is sensor technologies that allow devices to collect target signals and to analyze user performance and health conditions based on the data obtained.

According to the classification of properties of signals, sensors are also divided into different types based on their functions and their target signal. Chemical sensors and physical sensors are two types sensors with completely different detecting methods. Physical signals from both users and environments are of great importance, e.g., body temperature is always a vital mark of thousands disease monitoring, patient care. Physical sensors are mainly related to strain/motion sensors, pressure sensors and temperature sensors. Temperature sensor is also the most popular one in labs and market at present, such for blood transportation for medical concern [12]. Kinematic sensors including speed and acceleration sensor, vibration sensor, are paid mess attentions on for applications, e.g., $\mathrm{AR}, \mathrm{VR}$ and game use, while the heart rate monitor witnesses high popularity in market either by strain or by electrophysiology. Recent strain/motion sensors are developed to realize gait recognition [13], joint angle estimation [14], VR games applications [15], fall detection [16], activity recognition [17], consumer sport application [18], etc.

Certain chemicals from bio-fluids (e.g., interstitial fluid, saliva and sweat [4]) usually contain key information of personal health condition and other important massages that play great role in disease diagnose and researches [7]. There are different types of analyte in each bio-fluid, among which glucose is the main target beside detection of other chemical bio-markers, including peripheral oxygen saturation, potassium and sodium ions and blood oxygen. And there are also lots of studies have been done to research biosensors, such as biochemical analysis [19], psychiatric disorders and symptoms [20], nurse [21], electrocardiogram monitoring [22] And eye health monitoring [23].

Technically, the working circle of any particular sensors that unveil the existence and quantity of specific physical quantities and chemicals relies on two basic steps including signal acquisition and signal transduction. The basic principle is simply based on the relation between parameters, which however is the key to signal acquisition as well as signal transduction.

Typically, the signal acquisition is mainly based on principles which link pa- 
rameter to be measured with another one, e.g., capacity, resistance and volume, which we already have simple measuring method for. For an example, a capacitive humidity sensor measures environmental humidity based on a change of capacitance between two detection electrodes [24] (see Figure 1), because electrostatic capacity of an object would change relevantly when change of ambient humidity occurs. Resistive humidity sensors and impedance humidity sensors also share the similar principle with capacitive humidity sensors as the resistance and impedance have relevant relation with humidity as well. The fundamental mechanism of signal acquisition is similar for every type of sensors, of which only details, e.g., relevant parameters, existent measuring methods of relevant parameters, environment, vary to meet specialized demand and recognition needs.

Chemical sensors also share the same basic principle. There is an example of chemical sensors, which demonstrates the signal acquisition process of NDIR Sensors. NDIR sensors are used in a gaseous environment to detect $\mathrm{CO}_{2}$ with infrared component according to characteristic absorption of itself. Generally, it consists of an infrared source, a tube, a filter and a detector. Once the gas is pumped into the tub, infrared source will generate infrared light of which the light of $4.26-\mu \mathrm{m}, 2.7-\mu \mathrm{m}$ and about $13-\mu \mathrm{m}$ wavelength would be absorbed. Based on that, the filter would select light at these wavelength after absorption, and the detector is then introduced to measure the amount of light going through the filter, which presents the density of $\mathrm{CO}_{2}$.

After acquisition of signals, a transduction is needed to convert collected data into information that we desire. In another word, the data collected are going to be visualized after further analyses. As mentioned before, the acquisition of signal is based on the principle, and it is also the mechanism for signal transduction where needs further analyses of details. Once the specific relationship of these parameters is found, the transduction is easily accessible with only few calculations.

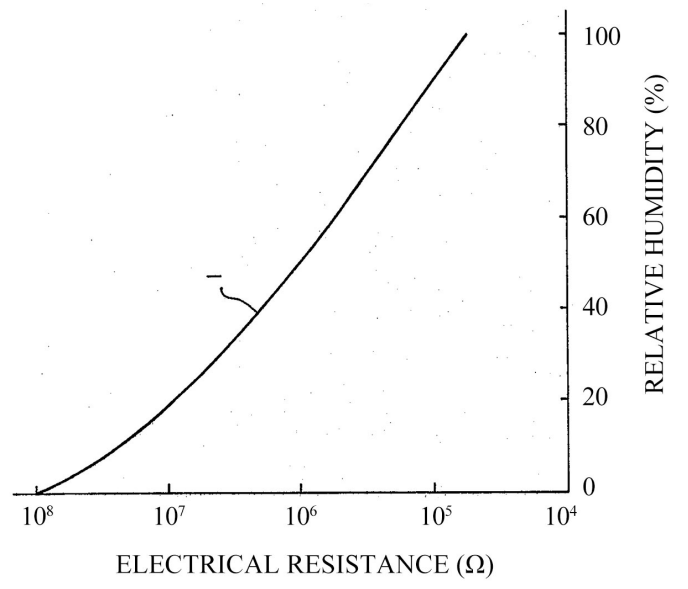

Figure 1. Changing resistance versus relative humidity of one type humidity sensor. Cited from ref [24]. 
As the figure shows, the resistance that consists in resistive humidity sensors has special relation with humidity. The humidity coefficient of electrical resistance at given temperature could be obtained by measuring the electrical resistance over a humidity range of $0 \%$ to $100 \%$, where specific formula could be obtained. With the formula and real-time resistance, the humidity could be simply calculated.

\subsection{Physical Signals}

Physical sensors are wildly adopted by wearable electronics to detect physical information, which are often designed for collecting data of both external and internal environmental, e.g., light, motion, temperature, magnetic fields, electrical fields, gravity, humidity, pressure, sound, stretch, motion of organism and position.

Kinematic sensors are widely adopted in motion detection for clinical diagnostics (movement disorders [25] [26] [27] [28] [29], neurological disorders [30]) and athletic performance monitoring [5]. This kind of sensor often integrates strain-based modulo to carry out multi-dimensional motion monitoring. The slight change of capacity, resistance and other relative parameters could demonstrate the deformation, strains, vibrations and applied pressures through carefully developed algorithm that designed and proved in lab. Wide definition of kinematic sensors also includes speed, acceleration, vibration sensors and gyroscope. They are in common use for visual reality (VR), augmented reality (AR) and other game equipment.

Temperature sensor is under wide development, especially the thermometer sensor, which is vital in clinical monitoring. Abnormal body temperature typically means the failure of body mechanics and should maintain between $36^{\circ} \mathrm{C}$ and $37^{\circ} \mathrm{C}$, a really narrow range. Measurements of temperature typically are reflected by the changes in the resistive, semiconducting, or optical properties of a material. These kinds of thermometer are often attached to skin and do local, spatiotemporal measurements. However, core body temperature is raised to demonstrate the global measurement of the body temperature against local, spatiotemporal patterns [31].

Tactile sensors are common type of physical sensors, which can measure information caused by physical interaction. Biological sense of cutaneous touch is the key principle for tactile sensors to detect stimuli resulting from mechanical stimulation and temperature. Tactile sensors are now wildly used in robotics and computer hardwares, especially in touchscreens.

Pressure sensors are also a category of physical sensors. For recently released iPhone, the realize of 3D-Touch utilize one type of pressure sensors named strain gauge. Apart from the tactile sensors of touchscreen, the stain gauge can detect the pressure change on the screen and output the signal about where and how much pressure has been changed, leading systems react to it and arouse the 3D-Touch interface. Pressure sensor array is also a hot field for physiological 
parameter monitoring and industrial process monitoring. At present, pressure sensor array is applied in invasive and noninvasive measurement of blood pressure as well as other body pressure. One kind of Electronic textiles is also invented based on pressure sensor array to monitoring both body pressure and external force.

Electrophysiological sensors are considerably popular in recent decades. The popularity of most advanced electrophysiological skinintegrated sensors is captured by heart rate (HR) monitor. The acquisition of electrophysiological signals typically relies on the simple circuit, e.g., RC-circuit. For specific applications, e.g., electrocardiography (ECG), electroencephalography (EEG) and electromyography (EMG), ECG detects the electrical activity of the heart by placing electrodes on the skin. These electrodes would detect the small electrical changes that are a consequence of cardiac muscle depolarization followed by repolarization during each cardiac cycle (heartbeat). And EEG measures voltage fluctuations resulting from ionic current within the neurons of the brain. An EMG detects the electric potential of muscle cells when these cells are electrically or neurologically activated through comparing the potential difference (voltage difference) between a pair of electrodes. In commercial monitors, ECG is more widespread with a set of gel-based electrodes attached to skin which enable ECG monitoring with well-established instructions, such as determination of electrode pasting positions, signal frequency, data acquisition and analysis. Because ECG has large amplitude $(1 \mathrm{mV})$ and its detection is much easier than other electrical signals, such as EEG and EMG.

Xiaomi, Apple and other well-known technical companies developed their own products that contained heart rate monitor, among which apple watch was wildly admired. These products, e.g., apple watch, xiaomi brand, Amazfit Watch, utilize different methods in HR monitoring, where apple watch adopts Digital Crown Electrode, Back Crystal Electrodes and optical sensor to monitor use's heart rate, Xiaomi uses PPG heart rate sensor instead. And in addition, apple also developed further applications of HR monitoring, e.g., they enable users to collect their ECG and they've built an algorithm to detect low, high or irregular heart rate, which was proved to possess extraordinary high consistency with lab results.

Environmental signals, e.g., light, gases, humidity, are also vital for both internal and external monitoring. UV radiation exposure [32] [33] 571,572 is of great importance for health alert of particular skin disease, such as ultraviolet allergy, and repair after surgery.

\subsection{Chemical Signals}

Chemical sensors are able to provide users with composition of chemical environment of internal bio-fluids and external environment, where vital chemical signals that indicate possible diseases and living performance are usually contained. Similar with physical signals, two-step theory (signal acquisition and 
signal transduction) is also pragmatic for most chemical sensing. In recognition processing, receptor will detect analyte (a certain chemical species) through a characteristic chemical reaction, then go into transduction step and output a signal as a report. This signal is in form of a measurable physical signal, level of which is related with the density of analyte.

Biosensors are one kind of chemical sensors, but they detect biological materials, e.g., tissue, microorganisms, organelles, cell receptors, enzymes, antibodies, nucleic acids, which will interact, bind or recognize with analyte. The processing of biosensors typically consists of a bio-recognition site, bio-transducer component, and electronic system. In bio-recognition step, biosensors will adopt bio-molecules from organisms or receptors to interact with analyte. This interaction can be measured by bio-transducer which then output measurable physical signals. In a biosensor, the bio-recognition site is named bio-receptor, where the bio-recognition step will be completed. According to types of bioreceptor interactions and bio-molecule used, bioreceptors can be classified into antibody/ antigen bio-receptor, enzymes/ligands bio-receptor, nucleic acids/DNA bio-receptor, cellular structures/cells bio-receptor, or bio-mimetic materials bio-receptor. Moreover, among all the parameters, selectivity is the key factor of bio-receptors, which present the ability of bio-receptors to find analyte from a matrix of other chemical materials.

Similarly, biosensors can also be classified by types of bio-transducers, among which the most common types are electrochemical biosensors, optical biosensors, electronic, biosensors, piezoelectric biosensors, gravimetric biosensors, pyroelectric biosensors. Biosensors are wildly used in biotechnology, agriculture, food technology, biomedicine fields and health monitoring, e.g., blood glucose monitoring [9], interferometric reflectance imaging sensor, food analysis, DNA biosensors, microbial biosensors, ozone biosensors, metastatic cancer cell biosensors.

For a biochemical sensor, the surface attachment of biological elements is as important as analyzing them. Basically, two methods are developed, of which the most simplest way is functionalize the surface, letting biological elements coat it for following process. Alternatively, entrapping elements by lattices, e.g., hydrogel and xerogel, can also realize surface attachment. For a specific example, antibodies are commonly adopted as a biosensor to detect pathogens and toxins, which usually coat optics during the detecting process. And fluorescence would amplify the signal produced by the interaction between antibodies and analytesFor a biochemical sensor, the surface attachment of biological elements is as important as analyzing them. Basically, two methods are developed, of which the most simplest way is functionalizing the surface, letting biological elements coat it for following process.

Bio-sensors are capturing increasing attention around the decade with their great potentials to assist researchers, doctors and users themselves to grow a superior insight toward the human diseases and living performance with conti- 
nuous, timely and accurate monitoring of living bodies. Bio-fluids, e.g., saliva, tear, sweat, interstitial fluid, are being wildly researched by many researchers. Sweat is under exploitation by Seshadri et al. to demonstrate a potential platform for athletes [5]. Tears contain numerous types of chemicals, of which a proportion are found to have certain correlation with eye illnesses. Driven by increasing focus on eye health, researchers have invested in the smart contact lens to carry eye health monitoring, allowing users to avoid dehydration and electromagnetism [34].

\section{Resent Applications in Wearables Fileds}

Wearable electronics are gaining increasing attention in recent decades as behavioral modeling, pattern recognition, medical monitoring are getting more popular both in research field and market. In these fields wearable computers are quite essential for data collecting from people's moves, surroundings and their physiological activities. They usually have sensory integration, with which wearable devises are capable to sensor and collect users' and environment's information, such as users' heart rate and step number, light conditions, etc. In the following process, computers would introduce specific arithmetic to output data in need, e.g., health conditions, sleeping record. And when compared to traditional equipment used to obtain these information which often have cumbersome hardware, matched computers and many wires, wearable electronics have absolute advantage for their smaller shape and portability. Though wearable electronics are confronting challenges and restrictions at present, numerous promising sensors and platforms are developed in labs, hooking the attention of researchers and companies. Due to the wide feasibility of sensing technology, these appealing new sensors and platforms are exploited under heterogeneous fields and application needs. This part selects a few sensors and platforms of excellent latent in different emerging fields, and generates an overall view toward these potential sensors and platforms.

\subsection{Smart Contact Lenses}

As the electronic devices dominate the society, everyone is exposed to the screens and the rate of people with eye-concerns have dramatically surged due to unhealthy eye-using, including continual use of eyes without breaks, excessive exposure to screens. Besides, the age-related eye concerns are also gaining increasing discuss, including macular degeneration, cataract, diabetic retinopathy, and glaucoma. To equip people with clearer understanding of their eye-related health conditions, and offer instant alarm when eyestrain or pre-symptoms occur, an Eyes-health monitoring and alarm system is therefore highly required. Several kinds of eye monitoring sensors have been developed, such as smart contact lenses [35] [36] [37], eye tracking monitor [38], eye health monitor [23]. Bingbing Gao et al. demonstrated a type of sensor that attached to user's eyelids and carry out fluorescent analyses based on the fluorescent property of one special materialsinverse opal carbon (IOC) [23]. Smart contact lenses are more popular 
and possess much more deep research works. Smart contacts now evolve into many branches, including Retinal disorder diagnostics, intraocular pressure sensor, Glucose sensor [34] [35] [37] [39] [40]. For an example, Kim described one type soft contact lenses that integrate glucose sensor and intraocular pressure monitoring [35].

Compared with traditional contact lenses, smart contact lenses not only possesses the brand-new sensing technology, but also ought to have great transparency, stretchability, highoxygen and water permeability is also vital for smart contact lens, instead of lens-shaped polyethylene terephthalate or PDMS substrates. The traditional method for glucose detection has its intrinsic limit that it can only offer temporary data rather than real-time values by finger prick method [41]. To develop a pain-free and convenient alternative to the existing approach, Joohee Kim et al. adopts FET-based sensor which consists of the graphene channel and hybrid S/D.

When it comes to the analyte of smart contact lenses, Intraocular pressure is one main factor in the pathogenesis of glaucoma [42], which eventually leads to the loss of vision. For intraocular pressure monitoring, the inductance and capacitance in the RLC circuit of the resistance-based glucose sensor is utilized. High intraocular pressure would increase the corneal radius of curvature, which would in turn increases both the capacitance by thinning the dielectric and the inductance by bi-axial lateral expansion of the spiral coils. By detecting deformations of the eyeball, the two sensing-resistive strain gauges would send the data into a microprocessor which would then send back an output signal proportional to the contact lens strain. Therefore, by calculating the deformation of contact lens, the intraocular pressure could be obtained indirectly [37]. Base on this property, intraocular pressure is able to have a real-time monitoring.

Furthermore, tear also contains a wide variety of biomarkers for keratoconus (and keratopathy), trachoma, thyroid-associated orbitopathy, glaucoma, diabetic retinopathy (DR), systemic sclerosis, cystic fibrosis, cancer, multiple sclerosis, and Parkinson's the detection of ocular and systemic disorders, such as dry eye syndrome (DES), ocular allergies, keratoconus disease (and amongst keratopathy), others [36]. By measuring the presence of such biomarkers or metabolites from tears, corresponding disease progression could be simply monitored and early methods could be applied to provide best treatment for patients.

In ocular diagnosing, the system would take advantages of multiple bio-sensors embedded in it. Such as glucose sensor, glucose sensors based on the FET that consists of the graphene channel and hybrid S/D are adopted by Joohee Kim [35]. Glucose oxidase (GOD) [43] [44] would be immobilized on the graphene channel using a pyrene linker via p-p stacking. Atomic force microscopy(AFM) images confirm that the GOD would selectively binds to the surface of graphene channel. GOD in return catalyses oxidation of glucose to gluconic acid and reduction of water to hydrogen peroxide, which in the system is oxidized to produce oxygen, protons and electrons. Thus, the concentration of charge carriers in the channel, and the drain current, increases at higher concentration of glu- 
cose. The detection mechanism of glucose is illustrated in Figure 2 [35].

Except from censoring functions, the smart contact lens can be further equipped with shielding and dehydration protection [34]. Electromagnectic (EM) waves are known to cause eye diseases such as cataracts, and many products have already developed to protect eyes from EM waves, such as anti-blue ray glasses, anti-blue ray screen film. Sangkyu Lee et al. have demonstrated one possible methods to screen out EM waves and dehydration by a graphene-coated contact lens. When the graphene is exposed to EM waves, the electrons in orbital motion induce oscillating magnetic moments in response to the external magnetic field. Therefore, EM energy would be absorbed and turned into thermal form.

Though the multi-functional sensor is not yet developed, the multiplexed contact lens sensor mentioned before indicates substantial promise for next-generation ocular diagnostics, and there are also other contact lens exist, which have varied functions, such as electromagnetic interference shielding, dehydration protection, recording of electroretinogram [34].

\subsection{Internal and External Workload Monitor}

Athletes are under great pressure and often face excessive training and fatigue in order to optimize their performance, which however could backfire and even increase the potential for injury. As the paradox between heavier training aimed at better performance and low injury rate, an innovative and advanced technology is in require for sports field to assist coaches, athletes and physicians to minimize the injury rate and reach better performance. And recent studies have shown the potential of wearable monitoring systems in continuous physiological data thus permitting the development of accurate treatment plans and player-specific training programs to potentially mitigate and alleviate injuries. The system demonstrated by Seshadri et al. basically focuses on monitoring internal and external workload of athletes including physical performance and physical statuss [5], enabling coaches, physicians and athletes themselves to obtain a further understanding of athletes' movements and status (see Figure 3). To provide valuable informations that help improve outputs of athletes, physical performance detection is divided into position and motion detection, impact detection and biomechanics detection, while physical status monitoring consists of heart rate and electrocardiogram detection, muscle oxygen saturation and sleep quality detection.

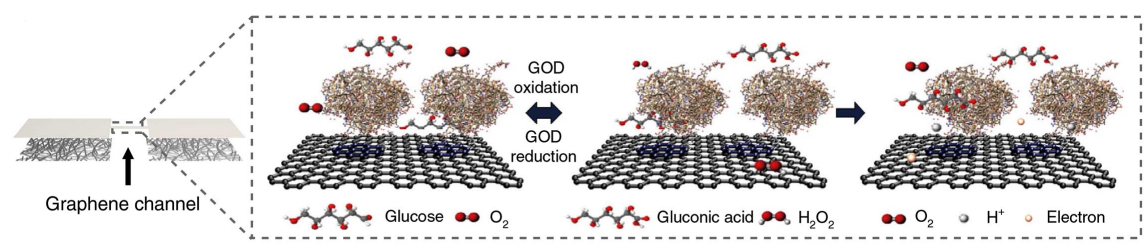

Figure 2. Schematic demonstration of glucose detection through the graphene channel. Cited from ref [35]. 


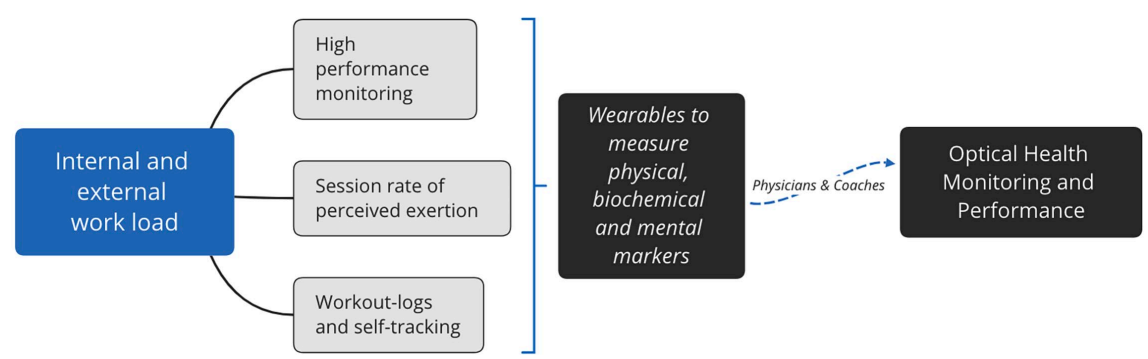

Figure 3. Simple system description of wearable workload monitoring. Draw by author of this article.

In position and motion detection, movementbased sensors, such as pedometers, accelerometers and global positioning satellite (GPS), are currently utilized for laboratorial and commercial monitoring. There are already several wristbased devices, such as Nike Fuelband, Jawbone UP, and Xiaomi Band, in the wearable sport monitor market, which use accelerometers to gain highly accurate analyses of movement with high sampling rates. Tri-axial accelerometers measure acceleration in three dimensions, and thus all physical activity can be captured and brought into analysis. Energy expenditure can also be calculated from tri-axial accelerometers [45] [46], which with data about position, movement and balance control during practices or games could help tailoring the training regimen of athletes to minimize the incidence of soft tissue injuries.

By introducing postulation of Banister et al. that athletic performance can be estimated as a function of fatigue and fitness, the author alert that the collection of data of athletes' physical performance and status allows coaches and physicians generating professional advices to maximize performance and minimize the potential of injury. In the theory, two forms of fatigue are included, named central fatigue and peripheral fatigue. Central fatigue is the fatigue resulting from the central nervous system (CNS) and the transmission of signals from the brain to the muscle. Peripheral fatigue is the failure to maintain an expected power output caused by the depletion of glycogen, phosphate compounds, or acetylcholine within the muscular unit or by the accumulation of lactate or other metabolites that are released during activity. In this athlete sensing system, wearable sensors aim to measure indicative of peripheral fatigue of athletes.

In analyzing of fatigue and fitness, internal and external workload are significant data. Internal workload includes the session rate of perceived exertion (sRPE) and heart rate. At the completion of each training session, athletes provide a 1 - 10 "rating" based on the intensity of the session. The intensity of the session is multiplied by the session duration to provide the internal training load. The product can be thought of as the athletes' "exertional minutes". By linking external workload to the load placed on the body and using torso-mounted wearable devices to quantify, the GPS and the tri-axial accelerometer could obtain instantaneous rate of change of acceleration, and then get player load. The work load can also be divided into acute workload and chronic workload, which present 1-week period and over 3 - 4 week period load respectively. Gabbett 
suggested that the ratio of the acute-to-chronic workload (ACWR) can be used to determine if an athlete is overtraining, undertraining, or training at the opportune intensity. Furthermore, Gabbett showed that calculation of this ratio enables sports scientists to predict the chance an athlete suffers an injury as a result of improper load management. As the paper states, when ACWR $\leq 0.99$, the likelihood of injury for fast bowlers in the next 7 days was $4 \%$. However, when the ACWR $\geq 1.5$, the risk of injury was 2 - 4 times greater in the subsequent 7 days.

Based on this concept, there are two main methods to calculate the training load of the athlete, named RA model and exponentially weighted moving average (EWMA) model. RA model uses acute workload relative to 4-week chronic workload. The four main equations are listed below.

Exertional minutes per workout $=(\mathrm{SRPE}) \times($ duration in minutes $)$

Acute $\mathrm{PL}=\sum_{D=1}^{D=7}$ exertional minutes per workout

$$
\text { Chronic PL }=\sum_{W=1}^{W=n} \text { Acute PL } / n
$$

ACWR = Acute PL for given week/Chronic PL

The RA model argues that each workload in an acute and chronic period is equal, which means the relationship between load and injury is linear. However, the author point out that key drawbacks of this model are that the model does not account for any decays in fitness and it does not accurately represent variations in the manner in which loads are accumulated. Thus, EWMA model is put up to circumvent the drawbacks posed by RA model. Additional equations are listed below.

$$
\lambda_{a}=2 /(N+1), \text { where } 0<\lambda_{a}<1
$$

EWMA $_{\text {today }}=($ Exertional minutes per workout $) \times \lambda_{a}+\left(1-\lambda_{a}\right) \times \operatorname{EWMA}_{\text {yesterday }}(6)$

$$
\text { Acute } \mathrm{PL}=\sum_{D=1}^{D=7} \mathrm{EWMA}_{\text {today }}
$$

The EWMA model places a greater weight on the most recent workload an athlete has performed by assigning a decreasing weighting for each older workload value and the nonlinear nature of injury occurrence and workload.

And a recent study has investigated the differences between the RA and EWMA models pertaining to ACWR calculation and subsequent injury risk in elite Australian footballers. It turned out that the EWMA model had significantly greater sensitivity to detect increases in injury likelihood at higher ACWR ranges during both the preseason and in-season periods.

However, there are questions about the variability of GPS data and accelerations of the torso by monitoring the loads of the lower limbs. Because distance traveled and velocity do not represent the mechanical load experienced by the musculoskeletal tissue in games where players experience high loads of physical 
stress by performing explosive jumping and landing activities, which are not accurately captured by distance, speed, or torso athlete movement analysis systems, such as basketball.

Relating to accidents might happen during practice and competition and absence of any sort of "protection mechanism" in human, impact detection is developed to detect and eliminate chronic traumatic encephalopathy (CTE). CTE is a neurodegenerative disease found in individuals who have experienced repeated traumatic brain injury (TBI) or concussions. And in these conditions, stretching, compression, and shearing of axons during sudden brain movements over extended periods are hypothesized to cause axonal injury. Thus, as the author alerts "The high incidence of such injuries in athletes is of major concern in modern collision sports" Though researches on concussions and CTE is now be conducted, they are still rudimentary and primarily supported by clinical models. Besides, there remains a strong clinical need to develop devices, which could quantitatively and qualitatively measure impact forces on the brain to decrease the onset of concussions and reduce the incidence of CTE. Recently, custom personal equipments, such as helmets and mouthguard, are being under research. For an example, Stenger et al. and McCrory et al. presented the potential applicability of mouth guards towards preventing head and spinal injuries in their research. Companies such as il Biometrics, Mamori, and Force Impact Technologies have developed mouthguards that can monitor concussions. Force Impact Technologies embedded sensors in their mouthguards, which enables relating collision intensity by color-coded LED's (green, blue, or red) placed at the front of the mouthguard. The colors are representative of the impact force delivered. A mild impact is presented by green, blue shows a medium impact force, and red represents a significant impact force. Bluetooth then would be used to translate information to appropriate medical personnel to initiate the necessary protections. In addition, to meet the need for fabricate wearable sensors of collision detect, several companies such as Noggin, Q30 Innovations, and $\mathrm{X} 2$ Biosystems are focusing on this fields. Noggin is creating a protective skull cap, inside which a gel cap could not only generate friction to keep it in place, but reduce impact forces on the head. A dry moisture wicking fabric is also used to protect athletes from heat-induced injury. For Q30, they are inspired by the woodpecker designing a device that prevents the brain from moving within skull by clamping down on the jugular veins. And the XPatch Pro wearable sensor and X2 Mouthguard devices by X2 Biosystems are now the most popular devices in the sports community. They utilize an adhesive worn behind the ear to record head impact which could transmit to a sensors data management (SDM) on an electronic device. This sensor contains a tri-axial high-impact linear accelerometer and a tri-axial gyroscope to obtain six degrees of freedom. Besides, X2 Biosystems also developed analytical software named xSposure to link acceleration measurements with impact duration, ranked from 1 to 10 . Gadd Severity Index (GSI), head impact telemetry severity profile (HITsp) and generalized ac- 
celeration model for brain injury threshold (GAMBIT) are also acquired during the process. Sporting companies, such as Reebok, also carried out some studies in this fields. Reebok entails a partnership with MC10. The Reebok Checklight includes one or more accelerometers wired up with MC10's "stretchable" electronics which consist of ultrathin gold electrodes that match the contour of the body. In another study, there is a dry, textile-based nanosensor developed, which could detect early signs of TBI by continuously monitoring various neural behaviors indicative of the injury, such as drowsiness, dizziness, fatigue, sensitivity to light, and anxiety. The device system contains a network of flexible sensors woven or printed into a skullcap worn underneath a football helmet. Zigbee/Bluetooth wireless telemetry are used to relay the data from the sensors to a receiver and to a remote monitor. The system also included a pressure-sensitive textile sensor embedded underneath the helmet's outer shell to gain the intensity, direction, and location of the impact force. The other sensors works as an integrated network within the skullcap and included a printable and flexible gyroscope that measured rotational motion of the head and body balance and a printable and a flexible 3-D accelerometer that measured lateral head motion and body balance. Additionally, the device was outfitted with physiological sensors to detect pulse rate and blood oxygen levels.

Biomechanics detection like epidermal wearable sensors can play a key role in quantifying human movement and monitoring changes in joint mechanics as an alternative method of biomechanics detection, such as measuring body kinematics via motion capture systems such as optical, inertial or magnetic units (IMU), electrogoniometers, and mechanical tracking, because obstacles exist due to massive volume and lack of flexibility. "motusBaseball Sensor", the first wearable device approved by the MLB for ingame use, was developed by Motus Global to gain a better understanding of the factors that cause pigment damage. This wearable sleeve could quantify the strain exerted by a pitcher and also contains five sensors and an analytic program to qualify biochemical analyze. And a single sensor is placed near the elbow in order to measure the stress exerted on the UCL.

As a significant status of human, heart rate and electrocardiogram undoubtedly need constant monitoring. Heart rate (HR) is a key indicator of physiological adaptation, exercise intensity, and workout effort. Prior studies have concluded that HR data analysis enable researchers to more accurately quantify physical activity in individuals. In HR monitoring fields, transducer worn around the chest is now the standard device, which could store data locally for 1 - 2 weeks or transmit to a wireless wrist display alternatively. And new methods, such as photoplethysmography (PPG), using optical sensors to detect HR direct from wrist or fingertip by monitoring blood volume changes as a function of transmitted and reflected light. There are already commercial products in the market, including Xiaomi mi Band, Apple Watch, Garmin and Fitbit devices, Komodo AIO Smart Sleeve, Jabra Sports Pulse headphone, WHOOP Band, and 
the Zephyr Bioharness ${ }^{\mathrm{Tm}}$. The Komodo AIO Smart Sleeve utilizes a conductive liquid to connect two electrodes on the wrist and under the arm. The Jabra Spaots Pulse headphones, however, should be placed against the bottom of the inside rim of the left ear canal, and this "hearable" could produce result 99.2\% comparable to ECGs. Though a great number of methods have been developed to obtain HR information, their accuracy is still limited, which is supported by a study by Wang et al. where a number of wrist-worn heart monitors were compared to a gold-standard ECG. This study strengthen the concept that none of the wrist-worn devices could reach the accuracy of a chest-strapbased monitor. Besides, these devices were most accurate when measuring resting HR and often loss their accurate while exercising.

Additionally, a growing interest in epidermal electronics to monitor HR prevails in order to mitigate accuracy-related issues posed by wristworn devices. Kabir et al. developed an optimal system to provide the best agreement with the Frank orthogonal ECGs, which introduces the long-term monitoring of a vectorcardiogram (VCG). A VCG could present the movement of the heart vector in three orthogonal dimensions and offer complementary massages for a 12-lead ECG. And abnormal electrophysiological substrate with life-threatening ventricular arrhythmias and sudden cardiac death (SCD) could be diagnosed with the help of analysis of VCGs. And in a recent study, Lee et al. created a self-adhesive ECG patch that conformally laminated onto the wrinkles of the skin, maintained robust contact, and self-adhered onto the epidermis. This device records various bio signals from an ECG, EMG and electrooculogram (EOG) without skin irritation. There is another study with a multifunctional epidermal device which is capable of measuring biosignals from both ECG, EMG, and temperature. Sensors of this device directly contact the skin via an elastomeric stamp, and it is transfer printed onto the skin through the application of an acrylate/silicone spray-on-bandage. The gel-less electrodes also provided a more convenient and stable biopotential measurement platform when tested on a healthy human subject undergoing walking and running tests.

"Smart garments" is another emerging filed for HR monitoring with sensors into textiles. A wearable ECG monitoring garment of this device utilized electrodes from carbon-derived paste which was applied to the skin and dried for 5 min resulting in a flexible and detachable electrode. And an ECG was affixed to the garment and used to measure ECG signals during walking and running.

However, as the author asserted that multiple issues with HR monitoring exist: Firstly, the physical fitness, body composition or other personal variances could belie the maximum HR value resulted form the standard formula. Secondly, HR may be unreliable because of lack od extremely controlled environments. Thirdly, HR fails to provide specific information about the responds of the working part of the body. Thus, the author concluded that measurement of an HR value currently provides little to no use when athletic trainers want to quantify the workout of the athlete to determine the ACWR and to predict the 
incidence of soft-tissue injuries during the rigors of training camp or live performance.

Muscle oxygen saturation, which refers to the amount of oxygen in the blood of muscles, is a measurement that has emerged as a useful parameter to help athletes optimize their performance. But due to the lack of wearable device fabrication based on $\mathrm{SmO}_{2}$ monitors, it still remains an emerging area. Commercial wearables usually adopted light waves to measure the amount of oxygenated and deoxygenated blood in the muscles. The Moxy Monitor and Portamon devices can be manually strapped on to any muscle group and communicates with portable devices through Bluetooth and a custom app. It uses optical techniques to obtain oxygenated hemoglobin concentration $\left(\mathrm{HbO}_{2}\right)$ and deoxygenated hemoglobin concentration $(\mathrm{Hb})$ in the muscle during exercise by shining nearinfrared light (NIR, $0.7-1.4 \mu \mathrm{m}$ wavelength) into the muscle and by detecting reflected light. There are several benefits to use muscle oxygen saturation $\left(\mathrm{SmO}_{2}\right)$, for it measures localized muscle performance, and could determine whether the working muscles are beyond their limits to inform the athlete that their muscles are running low on oxygen and they cannot sustain the current activity, and could also enable evaluating muscle recovery, all of which could definitely help trainers to provide instrumental suggestions to maximum their performance.

But a key question left unsettled in the field. Can the integration and translation of $\mathrm{SmO}_{2}$ levels measured from wearable sensors into internal workload models (HR or sRPE) better monitor athletic performance and predict soft-tissue injury in elite-level athletes? And indeed, clinical validation of current devices is needed to enable this translation.

Sleep quality and duration is another important indicator of health and is known to influence the performance and recovery. Recent studies are focusing monitoring body movement patterns as a measure of sleep restfulness. Examples of wearable devices currently in the market which monitor and track sleep quality are the Fitbit sensors, Jawbone UP, Misfit Shine, Komodo AIO Smart Sleeve, Polar watches, and WHOOP band. The WHOOP band as the first wrist-based device could proactively prescribe the hours of sleep needed to guarantee a full recovery. Physiological markers (e.g., resting HR, HRV) are measured to present strain, optimize recovery, and maximize performance on a daily basis, with which the algorithmic platform could determine the physical exertion during workouts while using this data to estimate the length of sleep required for a full recovery. Various studies have a consensus that a lack of sleep lowered athletic performance, worsened lung function, decreased the time to fatigue, increased injury risk, and increased lactic acid production thereby increasing the likelihood of post-workout muscle fatigue and soreness. Nevertheless a new study came into a conclusion after its careful-controled experiment that no device is able to accurately captured activity information through a whole day. Thus Polysomnography (PSG) still remains the gold standard for sleep monitoring, and should be adopted in studies to quantify the function of wearable devices to 
track sleep.

Besides, monitoring internal and external training loads can also enable sports trainers and clinicians to assess the fatigue and fitness levels in real time. Internal workload includes the session rate of perceived exertion (SRPE) and heart rate, while external workload can be defined as the load placed on the body. Both of internal and external workload can be quantified using sensing system mentioned before.

\subsection{Smart Skins}

Human skin is not only a "skin" but also serves as a mirror of internal organ functions, which consists of epidermis, dermis and subcutaneous fat from outside to inside. Skin offers a physical permeability barrier to offend against infectious agents, and also provides functions in thermoregulation, sensation, ultraviolet protection, and wound repair and regeneration. Besides, skin contains blood vessels, lymphatic vessels and nerves linking to the remote organs. As such valuable information available from the skin through visual inspection and palpation, Someya et al. asserted "the skin is the most practical organ" with "tremendous potential to non-invasively monitor different biological signals from multiple organs" [6].

As mentioned, skin is a complex organ of human and indicator of many informations towards physical health and potential diseases. Thus, to better understand skin in research realm, to create new possibilities for continuous monitoring of fitness in commercial arena and to transform the provision of healthcare and methods of patient monitoring, smart skins are developed, providing devices in close contact with real skin and enabling monitoring minute physiological changes that occur very gradually over long periods in daily life. In recent years, smart skins witnessed a surge in its development, such as the achievement of skin electronics of higher mechanical deformability, robustness, improved skin compatibility and higher device density [47].

Smart skins in this paper focus on human physiology, phenotypes that lead from health to disease and continuous monitoring of fitness and sleep for consumers.

The signal acquirement of this sensing system are generally divided into three typical types, including electrical signal acquirement, physical signal acquirement and chemical signal acquirement.

Electrical signal measuring includes heart monitoring and brain monitoring by electrocardiography (ECG), electroencephalography (EEG) and electromyography (EMG). ECG detects the electrical activity of the heart by placing electrodes on the skin. These electrodes would detect the small electrical changes that are a consequence of cardiac muscle depolarization followed by repolarization during each cardiac cycle (heartbeat). And EEG measures voltage fluctuations resulting from ionic current within the neurons of the brain. An EMG detects the electric potential of muscle cells when these cells are electrically or neu- 
rologically activated through comparing the potential difference (voltage difference) between a pair of electrodes [48]. In commercial monitors, ECG is more widespread with a set of gelbased electrodes attached to skin which enable ECG monitoring with well-established instructions, such as determination of electrode pasting positions, signal frequency, data acquisition and analysis. Because ECG has large amplitude $(1 \mathrm{mV})$ and its detection is much easier than other electrical signals, such as EEG and EMG.

Temperature, pressure and strain are three main physical signals monitored by smart skins. As a basic indicator for health status, body temperature could be obtained through sensors embedded in smart skins, provide real-time monitoring for clinic purpose. Platinum Thermistor (PT100) is one type of temperature sensors, which has a calibration circuit and a high precision gain amplifier with gain of 10. A wheatstone bridge circuit is embedded to read temperature between $0^{\circ} \mathrm{C}$ and $40^{\circ} \mathrm{C}$ corresponding to $0-1 \mathrm{~V}$, by placing the sensor in one arm of the wheat stone bridge circuit [49]. Pulse and blood pressure could be derived from ECG and PPG waveform [23]. Pulse and blood pressure are signals that reveal cardiovascular status of users, with smart skin sensing system, these physical signals are easily accessible, simplifying self diagnose and assisting clinician in analyzing.

In the context of strain, various body motions can be recorded as indicators, including tremor, another clinically relevant measurement. A simple-structured and low-cost graphene woven fabrics (GWFs) strain sensor is now able to distinguish different strain levels of human motion by the changes of current pathways and resistance cased by high-density cracks [50]. And in recent years, optical fiber strain gages are also developed with better fatigue behavior [51].

Aberrant or altered body motions are also important indicators of some neurological disease, which could be discovered and given timely treatments with the skin sensors. Besides, the locations of physical impairment and tremor conditions could also be detected by smart skins to infer the affected region of the brain.

For chemical signal sensing, glucose is the main target beside detection of other chemical biomarkers, including peripheral oxygen saturation, potassium and sodium ions and blood oxygen. Without painful blood sampling and scarring of the pinprick sites, this non-invasive wearable sensors could monitor glucose in interstitial fluid using needles, which have been actively studied and commercialized in recent years. Moreover, recent researches have shown the possibility of simultaneous and selectively measurements for multiplexed in situ perspiration [52], with which significant indicators, such as glucose and lactate, could be obtained in a more easy way. Electromagnetic sensing and Fluorescence technology are two main methods in glucose monitoring. Glucose concentration changes would cause variation of the dielectric parameters of the blood which electromagnetic sensing uses electric currents to detect and provide glucose analysis. Measuring changes in fluorescence resonance energy transfer between a fluores- 
cent donor and an acceptor and measuring glucose-induced changes in intrinsic fluorescence of enzymes are two types of fluorescence technology for glucose detection [9]. Other physical status, such as muscle fatigue, is accessible as well by adopting smart sensing system. Peripheral oxygen saturation is available by optical methods, such as perfusion index, near-infrared spectroscopy, laser-Doppler flowmetry, and orthogonal polarization spectral [53]. What's more, various environmental and psychological factors are also obtainable with directly contact devices, offering detailed physiological parameters to help building correlation between specific parameters and health conditions, such as relation between transepidermal water loss and skin barrier function and relation between skin surface $\mathrm{pH}$ and different environments.

However, there are several drawbacks and challenges for smart skins. For physical detection, the temperature monitoring is limited in surface temperature rather than core body temperature which is the actual clinically important measurement. And in chemical detection arena, it is reported that the glucose levels in interstitial show a significant delay of approximately $20 \mathrm{~min}$ when compared with glucose levels in blood, which may damage the real-time of monitoring. Besides, the enzymes used in glucose sensors are prone to error for cross-contamination of the sample by other analytes. Because biofluids often contains many materials that would affect the accuracy of glucose oxidase detection, which is much more apparent at low sugar concentrations. Except these drawbacks, the sensor sensitivity and lag time for on-skin measurement are also under a need of improvement. As the glucose level in sweat is 100 times lower than that in blood. And to ensure robust and representative detections of blood glucose, sensors require high sensitivity and reliability.

What's more, for individuals with certain allergies, contact dermatitis is an important problem. And for long-term use, the possibility of causing allergies through cutaneous sensitization to metals and other materials used in skin sensors cannot be ruled out. Overhydartion and maceration of the stratum corner and interfere with its normal barrier function might arise for the low gas permeability that inhibit the secretion of sweat or other fluids from the skin. In addition, the non-physiological space created between the skin and the electric device creates a challenge in measuring a biofluid such as sweat. The factors result in the delay or low sensitivity of skin surface measurements compared with those of core body or blood should be optimized and calibrated individually. Another challenge is data analysis. The environment noise, body noise, motion artifacts and sensor misalignment might hide faint biological signals. Thus, filtering techniques and superior algorithm are needed to extract the desired biomarkers from clean data. Besides, the indirect measurements may face a great risk of artifacts than traditional tests of clinically validated biomarkers as wearable sensors often need calibration and crossvalidation through the detection of multiple different markers. 


\subsection{Smart Wearable Sensors for Agriculture}

As the demand and human population grows, the crop productivity require a $60 \%-100 \%$ increase from 2005-2007 levels. However, a major crop losses happened worldwide due to the impairment of environmental and biological stresses and growth and yield. Besides, microbial pathogens and virus-related disease also contributed to the crop decrease. How to manage the plant stress-related events in a changing climate is now the major challenge for agricultural productivity, which depends on more precise management of limited resources and costly agrochemicals. Thus an innovative and convergent technological method for agricultural managements is required to increase total productivity. Recent studies have presented the potential of nanotechnology to reduce environmental and biological stress via optimizing the use of limited resources. And in their paper, Giraldo et al. presented a nonabiotechnology-based sensing system where wearable sensors are utilized in part of the smart plant sensing systems [1]. To monitoring internal and external informations of target plants remotely, two main types of sensors, including optical nanosensors and wearable sensors, are adopted.

In traditional methods of genetic modification, gene gun particle bombardment and agrobacterium tumefaciens are in wild use. Gene gun is also known as "biolistics", accelerating hightensity particles to the appropriate velocities which enables them penetrate barriers such as cell walls and be propelled in to the target cells [54] [55], Figure 4 illustrates the processing of Gene Gun.

Agrobacterium tumefaciens is a soil borne bacterium that is able to transfer part of its genome to infect plants. It has the ability to penetrate into cells and integrates stably its genetic materials into the plant chromosomes. Based on this character, researchers could rebuild genome of agrobacterium tumefaciens before infection to ensure the target gene finally be integrated into the plant chromosomes and successfully expressed in cells [55] [56] [57]. However, these two methods highly require genetically amenable plant species, which actually hinders the researches into allkinds plant. Thus, sensing systems for crops have been limited by few accessible transformation methods and plant species. In this paper, nanomaterial-enabled methods are introduced as an alternative way to satisfy the universal of DNA transformation for wild-type plants. Singlewalled carbon nanotubes (SWCNT) coated with DNA are able to penetrate lipid bilayers in a passive and spontaneous way.

Thus transfection of DNA is available if bind plant expression cassette to SWCNTs and application to leaf lamina by a needleless syringe [58] (see Figure 5). Although alternative approaches have been developed, how nanomaterial and plasmid properties determine transient versus stable gene expression in plants still require further exploration.

Once the nanosensors successfully expressed inside cells of target plant, the most significant characteristic of optical nanosensors fluorescence-is introduced to transfer biochemical signals. As the paper says that Nanomaterials have 

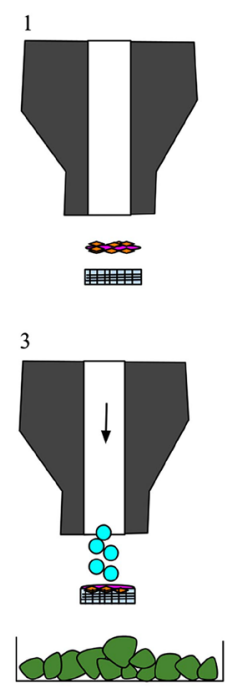

Figure 4. Schematic illustration and principle of a biolistic particle delivery system that injects cells with genetic information. "Gene Gun" by RachelBrooks15 is licensed under CC BY-SA 4.0.
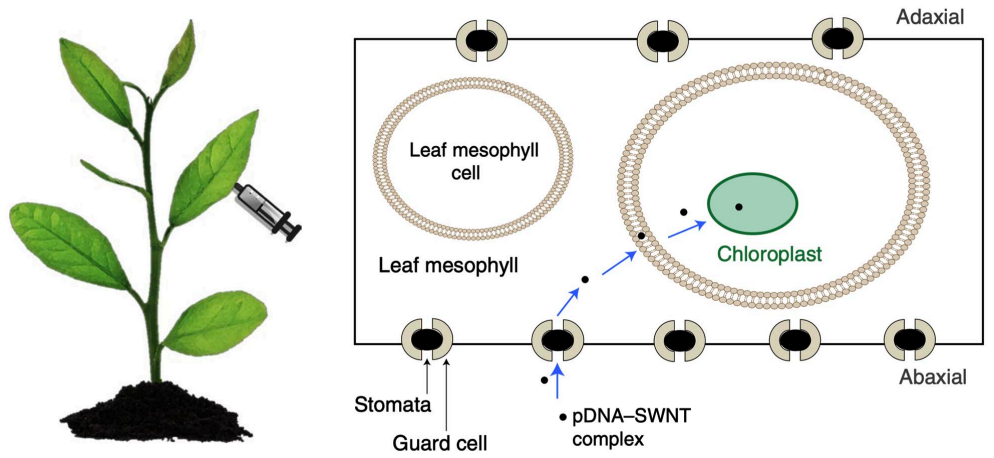

Figure 5. Simple demonstration of pDNA-SWNT complexes' entering into the leaf mesophyll through stomata pores. Cited from ref [58].

ultra-low photobleaching, fluorescence in lower transparent-background windows of living tissue and allow the detection of analytes with high spatiotemporal resolution down to singlemolecule level and millisecond timescales. As nanomaterials have protein-protein interactions or covalent bond modifications, their fluorescence intensity or wavelength in visible range of electromagnetic radiation spectrum would vary relatively. With fluorescence imaging devices, optical nanosensors could provide fluorescence imaging regarding to specific nanomaterials, allowing high spatial and temporal resolution for plant's monitoring. Near-infrared (nIR) is one of the optical technology. As every chemical species has its unique nIR absorptions, and based on this intrinsic property of the species, the chemicals could be detected by carefully-designed instruments which often contains a source, a detector, and a dispersive element to create nIR waves environment, and allow the intensity at different wavelengths to be recorded in the spectrum form. When compared with the nIR absorption spectrum of specific chemical, analyses could be introduced to determine the presence and con- 
centration of this chemical. And there is also near-infrared fluorescent materials which however emits specific nIR waves, such as SWCNT mentioned before. And in this case, the instruments are much simple that only a sensor or detector is deeded to record the emission. Besides, quantum dots (QDs) are also widely used nanoparticles for optical sensing applications, and they are fluorescent nanomaterials with bright and tunable emission range from the visible to the nIR. In addition, QDs could allow facile modification of structural and surface chemical properties as well.

However, current optical detection by embedding nanosensors in plants confronts low signalto-noise ratios when compared to lab conditions. Moreover, it also lack of targeted localization in specific plant tissues, cells or organelles. Three possible approaches are mentioned in this paper, including filed enhancement by gold nanoparticles, in situ synthesis of artificial sensors and coating nanosensors with targeting moieties. By coating viruses with a thin gold layer could keep the targeting capability of the virus. In situ syntheses rely on metal-organic frameworks(MOFs), fluorescent of which allows them to report acetone levels in air, avoiding the delivery of nanosensors to targeted tissues or sub cellular compartments. Alternatively, adopting targeting moieties such as peptides to coat nanosensors enables specific localization in plant sub cellular compartments.

This system also utilizes wearable nanotechnology-based sensors. Flexible wearable nano electronic circuits are implanted on plant surfaces to enable wireless communication of low concentrations of volatile molecules in Real time. Wearable SWCNT-graphite sensors are available to radio frequency (RF) for wireless monitoring without power consumption. Moreover, wearable nanotechnology-based sensors have certain elasticity that allow sensors to be bent on organisms with a radius of curvature as small as $100 \mathrm{~m}$. Electronic devices, such as smartphones and hyperspectral imaging cameras, can build direct communication with sensors in plants. Nanobiotechnology-based approaches enable transferring invisible plant stress-related signals into optical, wireless or electrical signals that can be recorded by intellectual computers.

Overall, the optical detection is not limited to a certain analytes, as key signalling molecules of plant health could be given real-time monitoring, including oxygen species (ROS), calcium $\left(\mathrm{Ca}^{2+}\right)$, glucose, sucrose, nitric oxide (NO) and plant hormones such as abscisic acid (ABA), jasmonic acid, methyl salicylate and ethylene, which all represent the health condition of plants and harbinger of a certain disease.

For ROS detection, indicators are often small molecules/fluorescent dyes. ROS detection often depends on oxidation of the ROS probe itself. These dyes are usually stable, but upon oxidation by ROS, they would be converted into fluorescent materials because of the formation of resonant moieties. And with optical devices, such as nIR, the quantification of ROS could be ultimately provided. The wild-use indicators include Dichlorodihydrofluorescein diacetate (DCFHDA), 
Dihydrorhodamine 123 (DHR), N-Acetyl-3,7-dihydroxyphenoxazine (Amplex Red), Dihydroethidium (DHE), Emerging alternative dyebased probes [59]. In $\mathrm{Ca}^{2+}$ monitoring, the dye Fura-2 with a Cairn micro photometer or a GenIV-intensified Pentamax-512 chargecoupled device camera allows Calcium Imaging in plants [60] [61] [62]. Glucose level could be measured using FRET nanosensors by a modified version of a previously described method [63]. And sucrose flux is able to be quantified by the sucrose sensor, such as FLIPsuc-9001 [64]. DAF dyes have been very widely used by plant NO scientists to reveal likely sites of NO generation. NO can be visualised via fluorescence microscopy [65]. For plant hormones, acids and other health-related components such as methyl salicylate and ethylene, there are also methods with high sensitivity and selectivity. Liquid chromatography-tandem mass spectrometry (LC-MS) has been applied to the analysis of ABA in plant [66]. Chromatographic, spectroscopic and some bioanalytical means such as radio immunoassay (RIA), enzyme-linked immunosorbent assay (ELISA) and HPLC-ECD method were also reported for analysis of jasmonic acid [67]. Vapor-phase extraction and gas chromatographypositive ion chemical ionization-mass spectrometry are employed in salicylic acid analysis [68]. For methyl salicylate, MeSA were detected and quantified by fluorescence detection at an excitation of $292 \mathrm{~nm}$ and an emission of $360 \mathrm{~nm}$ [69].

In combination with digital software platforms, nano sensors and wearable sensors can monitor and measure the plant's health condition and could provide substantial advice for agronomic decisions on crop performance. In the future, these sensors need to equip with high sensitivity and high signal-to-noise ratios. Besides, smart nanobiotechnology devices on plants has not been valid outside of laboratory, accompanying the lack of applicability, accuracy and durability under crop field conditions. In addition, replaceability and reparability of smart nanobiotechnologybased sensors need more practical, efficient and scalable interfacing methods of plants with nanomaterials. It is suggested by the paper that experiments under real agricultural conditions should be carried out to test their performance when weather, plant growth and development have significant influence. What's more, an integrative studies linking smart nanotechnology based sensing, plant stride, resource deficit simulations and signal analysis with actuation of agricultural devices should also be further explored.

\subsection{Augmented Reality and Visual Reality}

Another reason for wearables' popularity in recent decades is the hit caused by VR (Visual Reality) and AR (Augmented Reality). Google glasses and Oculus Rift have presented the fascinating application of AR and VR, which led a trend around 2014. People realized the relation between AR, VR and wearable electronics through the hit, and hundreds of worldwide companies began to focus on wearables technology.

VR systems often utilize headsets or multiprojected environments to generate experience of a digital world modeled by developers. VR headsets are now the 
spotlight of developers for their portability. Similarly, these headsets, such as Oculus Rift, consist of two separate monitors and audio systems, but typically they will also be quipped with positional and rotational real-time head tracking for six degrees of movement to detect users' moves and react in the digital world, e.g., changes of scenes, different moves of virtual characters.

Augmented reality is another types of visual reality. Equipment of AR, such as google glasses, could enhance the real objects by computers. Google glass is actually an optic head-mounted display with the shape of a pair of eyeglasses, which consists of a touchpad, a camera and the display. Focusing on the realize of AR system of google glasses, a liquid crystal on silicon, fieldsequential color system and LED illuminated display are adopted to create light into wearer's eyes, which presents interface of electronic system right upon what user sees.

Technically, mixed reality is a merging of real world and visual world, which could generate a brand new world for users where visual and real objects can co-exist. And AR is actually one kind of mixed reality, which excludes real environment and virtual environment and include all different level merge of real world and visual world.

\section{Achievement of Stretchable Electrodes}

\subsection{Synthesis and Engineering of Materials}

With mass interest in wearable electronics, researchers realized the great potential of wearables as well as the limits of them at present. The need of stretchable electrodes arises because the incompatibility of traditional electrodes and wearable needs. Until recent days, many promising materials have been reported to have potential to create next generation of electronics. They include Carbon Nanomaterials, Conductive Polymer and Metal Nanostructure. Thought all of them possess specific advantages in electrodes making, defects also exist, becoming the main obstacles of realization of desired stretchable electrodes.

For carbon nanomaterials, the main members of this group in this filed are Carbon Blacks (CB), Carbon nanotubes (CNT), and graphene, all of which have extraordinary electrical and mechanical properties. CB has high electrical conductivity (about 25 at a packing fraction of $0.3(\mathrm{~S} / \mathrm{cm})$ ) [70] and commercially available characteristics [71], however, the processing of carbon black have a main disadvantage which would introduce hydrogen into edges of the carbon layers, and result in degradation of electrical conductivity. The one-dimensional CNTs owns superior intrinsic conductivity and mechanical characteristics, as well as high optical transparency, and thermal stability. A singlewalled carbon nanotube could exhibit high tensile strengths around $63 \mathrm{GPa}$ [72], and in theory, metallic nanotubes can carry an current of $4109 \mathrm{~A} / \mathrm{cm}^{2}$, which is more than 1000 times greater than those of metals such as copper [73]. However, the wide range of diameters of CNTs causes their electrical property varying from metallic to semiconducting. And also due to the impurity of CNTs, the resistance of them would be large. Two-dimensional structure of graphene realizes low contact re- 
sistance with organic electronic devices. But graphene oxide (GO) is electrically insulating, because of which the conductivity would suffer. Besides, it would also face cracks when stretching and cause a nonreversible degradation of the electrical performance.

Conductive polymer includes two promising materials that are polypyrrole (PPy) and PEDOT-based materials. The manufacturing of PPy is easy, and PPy also has great conductivity, good adhesion and is of non-toxicity as well. However, the electrodes made of PPy are of low sensitivity and unsatisfying conductivity. PEDOT-based materials exhibit high air and thermal stability and tunable conductivity $\left(10^{-4}-10^{3} \mathrm{~S} / \mathrm{cm}\right)$ [74]. But on the other hand, during stretching dried PEDOT:PSS film would suffer destruction due to the existence of hard segments and hydrophilic PSS chains. Besides, humid environment, however, would degrade its conductivity.

Traditional materials for electrodes also have their advantages in wearable electronics. Metal nanostructure is undoubtedly have outstanding conductivity and flexibility. Nano-wires (NWs), for an example, Ag NWs are also of great transparency [75] [76]. It is reported that Ag NWs have significant strength to guarantee the completeness of networks during repeated stretching. But, it is well known that $\mathrm{Ag}$ is relatively expensive in the market, which would limit its largescale producing. Though copper exhibits similar properties as Ag, the oxidation of $\mathrm{Cu}$ is still the main concern for Cu NWs [77] [78].

Nanoparticles (NPs) is another metal materials structure the show promising potential for stretchable electrodes. They need less cost while they are ecofriendly. The large scalable methods are also reported [79] [80] [81]. However, during stretching, unsatisfying sensing ranges and reliability occurs due to the irreversible breakage between the NPs.

To avoid main defects of one certain material, researchers have developed hybrid material which by combining two or more materials, introduces both advantages of their properties and reduces their weakness as far as possible. Taking advantages of their synergistic effects, many new potential conductive materials came into being.

Based on the intrinsic pros and cons of promising materials as aforementioned, I think there may be a way to combine the strengths of both carbon nanotubes and polymers. In theory, CNTs could achieve outstanding conductivity that is much better than metal. Besides, they exhibit superior tensile strengths, which could guarantee the integrity of system. What's more, they also have high optical transparency, and strong thermal stability, which makes them a great candidate for stretchable electrodes. Though the wide range of diameters and impurity result in large resistance and incompatible electrical property. I believe new scalable synthesis method would be developed to obtain purer CNTs with similar diameters, due to their wide use not only in stretchable electronics but many other fields. However, the flexibility of CNTs is unsatisfying, therefore, I suppose by combining polymer would undoubtedly increase the stretchability of 
CNTs electrodes. And it has been reported that $50 \%$ or even $80 \%$ deformation can be reached by combining CNTs and polymer, and it is enough for most wearable devices [82]. And the superior tensile strengths of CNTs could solve the problem of polymer-based electrodes as mentioned before.

\subsection{Designs of Stretchable Structures}

In order to achieve stretchability, there are two prevailing methods. The first method is to obtain stretchable electrodes and embed them in an elastomeric matrix (e.g., PDMS; polyester, PE; PU). And the other one is to utilize specific geometrical configurations, such as buckling, serpentine, netshaped, or springlike structures to crease stretchability [2].

To obtain stretchable materials, simultaneous blending of conductive materials and stretchable matrix is one popular method. It is a simple and scalable way via effective and large-scale mixing of conductive fillers and elastomeric matrix in solution or melting states. The increase of the ratio of conductive fillers could enhance overall conductivity, however reducing the stretchability of the composites. Besides, several problems need to be addressed. The surfactants used to enhance the compatibility of conductive fillers with matrix would also damage the conductivity. And most polymers are insoluble, which undoubtedly increases the difficulty of blending.

Constructing conductive network-embedding elastomer is another promising way to achieve stretchability without aforementioned issues. Elastomer-embedding approach is capable of achieving a myriad of promising characteristics simultaneously, such as a patterned sandwichstructured strain sensor fabricated by pouring the PDMS prepolymer (Sylgard 184) onto the Ag NWs line pattern [83]. However, challenge still exits. After the transfer printing process, the removal of the composites from the original substrates is significantly challenging. What's more, the constructed networks may hinder the infiltration of polymer, degrading stretchability.

Another approach was developed to address the low compatibility between building blocks and polymers. Elastomers-Constructing conductive network is to introduce conductive blocks on top of or within elastomers, such as aforementioned AgNWs based temperature sensor. Except depositing method, conductive blocks could also be infused inside the elastomers [84].

To acquire stretchability, utilizing structures, such as mesh-shaped or buckled structures, also have great success. The buckled structure is formed by relaxation of a restrained matrix, where uniaxial prestrain causes the linear buckles, and biaxial prestrain results in herringbone buckled patterns [85] (see Figure 6(a)).

CNT sheets wrapped in stretched rubber fiber cores have been realized by buckled structure and the resistance change of the fibers alters less than $5 \%$ for a $1000 \%$ stretch [85]. Besides, the conductivity could be further improved by reducing the diameter of the rubber core. However, buckled structure requires excellent adhesion between the conductivity blocks and the substrate, as well as 
complicated prestrain procedure, which is unavailable now for large scale manufacturing.

Another effective structure is Open-mesh structure [86] (see Figure 6(b)). The holes allow stretching via its reconfiguration to respond to strain from outside. Inspired from this, Zhu et al. have developed a wearable strain sensor by adhering the GWFs on PDMS and medical tape composite as picture shows [50].

Coiled structure can also realize stretchability. Can et al. and Sun et al. have both reported the effectiveness of coiled structure, fabricating a yarn-derived spring-like CNT rope with uniform, neat loops 120 [87] (see Figure 6(c)), and graphene-based composite fiber with "compression spring" architectures respectively 121 [89].

And better performance was observed than two dimension structure for the coiled structure could suppress the local stress formed in the conductive layer during the stretch. Moreover, the nonplanar motion of the coil could reduce the local maximum strain of the 3D coiled structure effectively.

It was reported the sponge structure has potential to construct stretchable networks. The porous sponge can deform to accommodate the strain under stretching. Yu et al. have declared a binary-network-structured PU sponge-Ag NWPDMS (PUS-Ag NW-PDMS) stretchable conductor with high performance [88] (see Figure 6(d)).

(a)

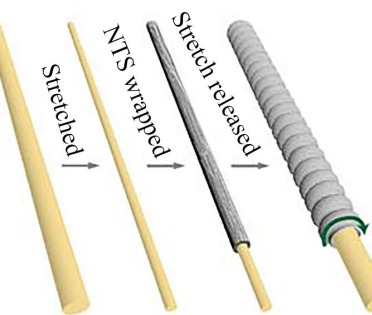

(b)

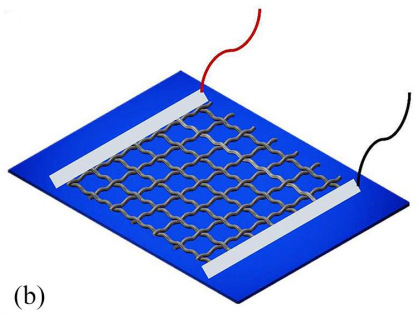

2

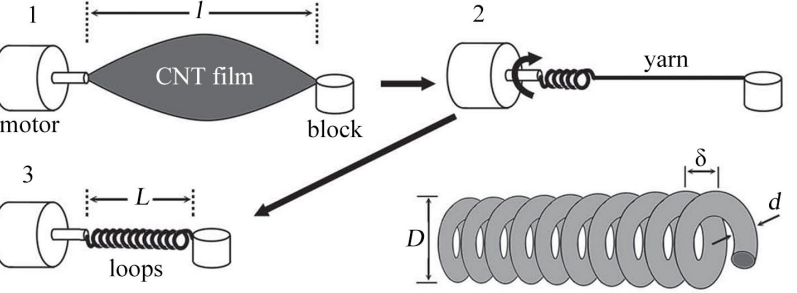

(c)

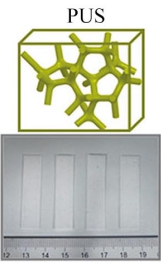

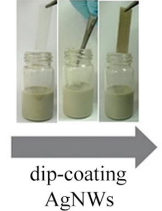
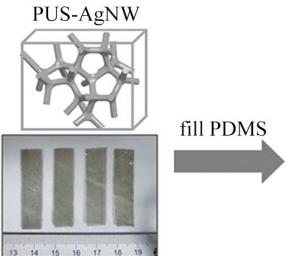

PUS-AgNW-PDMS

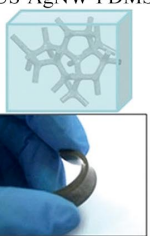

Figure 6. (a) Steps in fabricating a buckled structure material. Cited from ref [85]. (b) Schematic of a GWF-based strain sensor and top-view optical image of the open-mesh structure. Cited from ref [86]. (c) Spinning process for coiled structure materials. Cited from ref [87]. (d) Fabrication procedure of sponge structure. Cited from ref [88]. 
Except aforementioned stretchable structures, percolation network [90], serpentine [91] [92], and island bridge [93] [94] are also developed to achieve needed stretchability. Compared to designing stretchable materials, structure design has its priority for it won't result in unstable electrical functionality and mechanical integrity under large cyclic mechanical deformation [95].

\subsection{Promising Material: AgNW}

Silver nanowires (AgNWs) are 1-dimensional silver nanostructures with diameters that are typically in a range of $10-200 \mathrm{~nm}$, and lengths in a range of $5-100$ $\mathrm{lm}$. As an alternative material of traditional metal in circuit forming, AgNWs must be equipped with excellent electrical properties. AgNWs exhibit a relatively low sheet resistance ( $\sim 50 \Omega / \mathrm{sq})$, which also contributes to its high conductivity. However, several physical facts show that the conductivity of a nanowire will be much less than that of the corresponding bulk material. Because the edge effect will be quite significant. In other word, with the width of NWs decreasing, the mean free path to the wire width will be effectively shorten. And another problem is that the low sheet resistance would be an obstacle for signal distinguish. In order to use AgNWs for sensor constructing, the resistance level of the electrode is critical. If the resistance of an AgNWs based electrode is too low, the desired electrical signal discrimination ability would suffer. What's more, lowering the density of the AgNWs are also problematic because of unstable electrical contacts, though increases the resistance. However, compared to previous alternative materials, AgNWs still have significant conductivityr [96].

In wearable electronics and systems, the flexibility of electrodes are in substantial need to create close contact environment for sensing system. And high mechanical flexibility of AgNWs are observed by previous studies. And combining with different substrates, AgNWs can presents different flexibility. The rate of components of AgNWs based sensor also have great influence on its final mechanical flexibility [97] [98] [99] [100].

The thermal properties of AgNWs based sensors, such as temperature sensor, are also sensitive and stable [12]. As an aesthetic demand, the transparency of sensors also have its own attention ignorer to be invisible during daily use. And it is reported that AgNWs are also of great transparency [75] [76] [101].

In addition, AgNWs are now available through mass-produced method, such as solution based synthesis method [12]. Though AgNWs are competitive potential candidate with its excellent conductivity, great optical property, high mechanical flexibility, the poor adhesion with substrates becomes the significant obstacle toward their application [12]. Several studies have been carried out to embed nano-wires into polymer matrices, such as polydimethylsiloxane(PDMS) [102], polyimide [103] [104], NOA63 [105], and GFR Hybrimer [106].

Concerning with the oxidation and silver ion leaching of AgNWs, researchers are carrying out several studies, among which the Ag-Au coresheath nanowire presents potential compatibility. The inert gold sheath ensures the biocompatibility, preventing silver ion leaching which have adverse health effects on users 
[107]. Besides, the gold shell can also prevent oxidation of silver, which leads to the chemical instability of AgNWs. Cytotoxicity test and histological analysis also confirm that the Au sheath effectively improves biocompatibility by preventing Ag ion leaching and protecting Ag nanowires from oxidation [108].

The wildly used substrate for AgNWs are polymer, such as colorless polyimide (cPI) [104], poly (dimethylsiloxane) (PDMS) [102], NOA63 [105] and GFRHybrimer [106]. The conductive polymer is also a promising material for transparent, stretchable and highly conductive electrodes. They are often easy to prepare/produce, and of relatively high conductivity. Besides, some types of conductive polymer are proved to have non toxicity, such as polypyrrole, which is essential in wearables. And good adhesion is also declared by previous studies. What's more, excellent air and thermal stability, high transparency in the visible spectral region, tunable conductivity, etc., are also proved for some specific polymer.

For some specific polymer, they also posses electroluminescent properties which can be used to develop light-emitting devices. For an example, an elastomeric polymer light-emitting device (EPLED) is fabricated by Jiajie Liang. The EPLED is comprised by an electroluminescent polymer layer sandwiched between a pair of AgNWs based transparent elastomeric composite electrodes. It is reported to has high visual transparency, good surface electrical conductivity, high stretchability and high surface smoothness, all features essential to the fabrication of the EPLED [109].

However, there are many challenges for conductive polymer to apply as stretchable electrodes by some reasons. Sensors based on polymer, such as polypyrrole, exhibit low sensitivity and unsatisfying conductivity. And the existence of hard segments and hydrophilic PSS chains in several kind polymer would result in destruction of dried PEDOT:PSS film during bending and stretching, and the decay of conductivity with time under humid conditions [2].

To avoid main defects of one certain material, researchers have developed hybrid material which by combining two or more materials, introduces both advantages of their properties and reduces their weakness as far as possible. Taking advantages of their synergistic effects, many new potential conductive materials came into being. And sensors base on hybrid material are reported to have outstanding properties. Temperature sensor developed by Doo-Young Youn, Uihyun Jung, etc., adopted hybrid material which combined AgNWs and polymer. Desirable overall resistance, an acceptable sensitivity toward temperature changes, excellent mechanical stability and distinct linearity are achieved [12].

\subsection{Comparison between AgNW and Bulk Metals}

Silver is a common material used in high-tech devices for its electrical, thermal and other properties. And it is reported that silver exhibits the highest electrical and thermal conductivities among all metals [110] [111] [112] [113]. Since AgNWs are comprised of silver, here we carry out some comparison between 
bulk normal metal's and AgNWs' properties. In regard to conductivity, which should always be considered when fabricating circuit, silver and AgNWs possess the most excellent performance with their conductivity at $6.30 \times 10^{7} \mathrm{~S} / \mathrm{m}$ [114] [115], which as mentioned before is the highest among all the metals. The common used copper for wires held the second place with conductivity of $5.96 \times 10^{7}$ $\mathrm{S} / \mathrm{m}$, followed by annealed copper and gold with conductivity at $5.980 \times 10^{7} \mathrm{~S} / \mathrm{m}$ and $4.10 \times 10^{7} \mathrm{~S} / \mathrm{m}$ respectively. What's more, the most promising material for nanowires-platinum has $9.43 \times 10^{6} \mathrm{~S} / \mathrm{m}$, which is much less than silver and AgNWs. Though bulk silver's and AgNWs' conductivity derive from silver, there is a report that claims AgNWs possess higher resistivity than bulk silver does, due to electron surface scattering [116] [117].

The mechanical properties of AgNWs are wildly studied. And Xiaodong Li et al. have discovered that AgNWs have comparable hardness and elastic modulus to bulk silver [110]. They utilized nanoindenter method to measure the hardness and elastic modulus directly, where the nanoindenter monitors and records the load and displacement of the three-sided pyramidal diamond indenter during indentation with a force of around $1 \mathrm{nN}$ and a displacement resolution of about $0.2 \mathrm{~nm}$. The date obtained during the process, such as the load-displacement data, is able to calculate the hardness and elastic modulus of silver nanowires. The hardness could be obtained at the peak load as

$$
H=P_{\max } / A
$$

where $P \max$ is the peak load and $A$ is the projected contact area. The nanoindentation elastic modulus was calculated using the Oliver-Pharr data analysis procedure [118] as

$$
S=2 \beta \sqrt{A / \pi} E_{r}
$$

where $\beta$ is a constant that depends on the geometry of the indenter $(\beta=1.034$ for a Berkovich indenter) [119] and $E_{r}$ is the reduced elastic modulus that accounts for the fact that elastic deformation occurs in both the sample and the indenter. $E_{r}$ is given by

$$
1 / E_{r}=\left(1-v^{2}\right) / E+\left(1-v_{i}^{2}\right) / E_{i}
$$

where $E$ and $v$ are the elastic modulus and Poisson's ratio for the sample and $E_{\mathrm{i}}$ and $v_{i}$ are the same quantities for the indenter, respectively. For diamond, $E_{i}=$ $1141 \mathrm{GPa}$ and $v_{i}=0.07$ [118]. Ultimately, the hardness and elastic modulus values of the silver nanowires were measured to be about $0.87 \pm 4$ and $88 \pm 5 \mathrm{GPa}$, respectively, which match well with the nanoindentation results of the bulk silver single crystal [120] [121] [122]. The fluctuation of silver's hardness is due to the size effect. And they also found nanoscale hardness of silver nanowires is about 2 times higher than the macro/microscale indentation hardness of the bulk single crystal.

Besides, for further research of nanoindentation size effects due to strain-gradient plasticity, Minhua Zhao et al. proposed a study of strain-gradient plasticity 
that is based on characteristic material length scale, and nanoindentation is carried on single crystals of $\mathrm{Al}, \mathrm{Ag}, \mathrm{Ni}$, polycrystalline $\mathrm{Cu}$ and poly-synthetically twinned (PST) lamellar $\alpha 2$ - and $\gamma$-TiAl [122] (see Figure 7).

From the figure, we could draw an easy conclusion about the hardness of aforementioned six materials that silver has the second lowest hardness among these materials. In other word, single crystals of $\mathrm{Ni}$, polycrystalline $\mathrm{Cu}$ and polysynthetically twinned (PST) lamellar $\alpha 2$ - and $\gamma$-TiAl are harder than silver, while aluminum posses less hardness than silver does.

As the promising material for wearable electronics that have close contact with skins, the safe issue is always the spotlight. Silver has antibacteria property, while possesses great toxicity for human if gets into human body. Min Jung Kim et al. have carried out experiments towards toxicity of silver nanomaterials (AgNMs), exposing human blood to two different sizes of spherical particles (d 30 nm or $100 \mathrm{~nm}$ ) or nanowires ( $\mathrm{d} 40 \mathrm{~nm}, 1-2 \mathrm{~lm}$ in length) at a range of concentrations and incubation times and assessing deformability and aggregation of red blood cells. The result of this experiment showed that the rheological changes of red blood cells were mainly due to the Ag-NMs rather than the Ag ions [123].

In consideration of other metal nanowires, though gold nanowires have advantages over Ag nanowires in terms of biocompatiblity and oxidation tendency, their conductivity is lower than $\mathrm{Ag}$ nanowires, and producing long and thick gold nanowires in large quantity is very challenging [124] [125].

\subsection{Other Considerations of AgNW}

Though normal AgNWs suffer from Ag ion leaching, which equips AgNWs with both property of anti-bacteria and toxicity for human. Suji Choi et al. have reported a core-sheath method, that coats AgNWs with stretchable and biocompatible gold, have great potential to prevent total silver ion leaching besides with the oxidation problem of $\mathrm{Ag}$ [108].
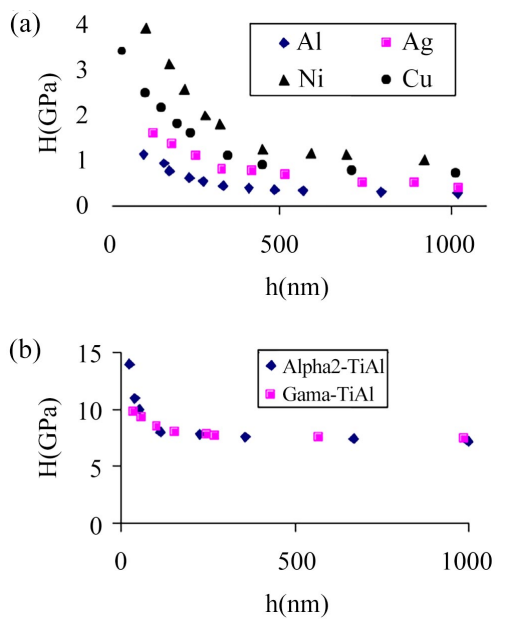

Figure 7. Hardness versus indentation depth of six different materials. Cited from ref [122]. 
Besides, wearable electronics are often used outside human body, the silver ion leaching and toxicity of AgNMs are much less effective in influencing human health, for which AgNWs are very promising material for wearable circuits' building. Along with its excellent conductivity, AgNWs stand out among various promising materials, e.g., AuNWs, CuNWs, silver nanoparticles, bulk copper, thus, AgNWs are material with great potential, and would be my choice for wearable electronics.

As for consisting structure, the sponge structure method is attractive in both stretchability and stability due to its special sponge-like structure. When comparing with other aforementioned approaches that generate stretchability, sponge method though contributes to less flexibility, the connection is much stronger than the others, leading to high stability of sponge-structure circuit. As Yu et al. had in their experiment, the resulting PUS-Ag NWs-PDMS conductors possessed excellent electromechanical stability even under high tensile strain (50\%) and a small bend radius $(1 \mathrm{~mm})$ [88].

They concluded the superiority of such sponge structure was contributed by strut rotation and bending, mitigating the accumulation of the strain in the individual struts. Later proved by Mechanical simulation, the 3D sponge structure could effectively release the strain on the metallic layer by transferring the strain deformation to rotation movements, preventing the occurrence of cracks. The stability is essential for a circuit, which decides the long-last of wearable electronics and their accuracy. For this reason, I would choose sponge structure method to build wearable electrodes or circuits.

\section{Challenges and Prospects}

Wearable devices are the significant trend for health monitor system, which would raise a revolution in heath-care field. For traditional assessment, the requirement of timeliness is often not met due to long time spent on collecting, sending and assessing. Moreover, the place that assessment take place is also limited in traditional way because of low portability of traditional equipments. Wearable computers are undoubtedly the best methods for health monitoring at present for the general aim of biosensors is to enable quick, convenient assessment at the point where the sample was procured.

Lots of researchers are now doing wearable technology and applications in medical monitoring. However, there are many challenges waiting for researchers. For an example, Jason Heikenfeld et al. are coming up with continuous access to analytes in biofluids [4]. By constant contact with biofluids, sensors could get instant informations from interstitial fluid, saliva and sweat. However, the filtration, active anlyte channels, $\mathrm{pH}$, salinity and other factors are still great challenges for sensors.

And the materials used for wearable devices also need further improvement. Heathmonitoring devices require good contact with users and great portability, for which stretchable materials that is easily shaped and of light weight is suita- 
ble for wearable computers. But there are still many works to do until suitable materials is developed. At present some kinds of stretchable materials are already be put into experiments, e.g." "Wavy" ribbons, 3D coil structure. Moreover, technology that print circuit on users' skin is also developed, but still need further improvement.

The life time, accuracy and steady sensibility of sensors are also waiting for development, especially for those indwelling sensors which would stay in users' body. During the transfer process, several types of deviations can occur, which include systematic errors and random errors. Bigger scale range, better linearity and sensitivity are required for the future improvement. And big effort should also be put into avoiding noise, which may result in random deviation of the signal.

Power is another challenge for wearable monitor that still mainly use batteries to drive the devices and have needs to change or charge the batteries frequently. Besides, batteries are often heavy and of big volume, which may damage the portability of wearable devices. Thus, a new power source is quite essential for the realizing of wearables. Recently, researchers have found several new methods for sustainably powering wearable electronics, e.g., triboelectric nanogenerator (TENG) [126], Serpentine-shaped batteries. The TENG can generate power for monitors by human motion, and Serpentine-shaped batteries are flexible and stretchable. These two types of new power sources present two main thoughts about how to create new power provider. TENG shows the idea that we should abandon traditional power and seek for new sources, such as bioenergy, while Serpentine-shaped batteries presents the thought that it is possible to make tiny and durable batteries. Literally, it is quite difficult to tell which thought would win in the future.

Apart from the technology, there are also a lot issues that wearable electronics caused in society. For a specific example, privacy is undermined when people's life is exposed to all these kinds wearable electronics. Google glasses have already faced this issues for they can photograph secretly, which undoubtedly annoyed people and raised society's privacy concerns. Consequently, the privacy issue became one reason for the stop of google glasses' selling.

\section{Market Overview}

According to IDC's report, in 2018172.2 million wearable devices are sold. And 46.2 million of then are contributed by Apple, which means Apple accounted for $26.8 \%$ of the wearable market, leaving other players far behind. The second company was Xiaomi with its 23.3 million units sold, which brought it $13.5 \%$ of the whole market. Then followed Fitbit, Huawei and Samsung with $8.0 \%, 6.6 \%$, 6.2\% market share respectively. Fitbit had sold 13.8 million units in 2018 and the amounts of devices that other companies sold were quite close, which are all around 11 million. These 5 companies have occupied $61.2 \%$ of the entire wearables market, leaving others to divide the left $38.8 \%$. 
Compared with 2017's market, these main players except Fitbit have witnessed different level of growth. Apple and Xiaomi have 39.5\% and $44.6 \%$ yearover-year growth respectively. Fitbit, however, had negative $10 \%$ growth, but still remained third with little more shipments. Huawei owned $147.3 \%$ yearover-year growth, which almost doubled its market share and made the company surpassed Samsung, although Samsung had $85.1 \%$ year-over-year growth which was quite significant. Others are reported to have $11.2 \%$ year-over-year growth, whereas, the market share is becoming smaller from $44.4 \%$ to $38.8 \%$.

Juniper Research said healthcare wearables market is going to reach $\$ 60$ billion by 2023, while industry analyst CCS Insight said Worldwide smart wearable device sales will double by 2022 , becoming a $\$ 27$ billion+ market. It is a huge difference between $\$ 60$ billion and $\$ 27$ billion, however, they are both telling that the wearables industry is still blooming in the following years. And there are some other trends in this market, which are expected to be more common in following years.

Smartwatches now occupies the major share, and would still be a hot. Consumers' daily requirements, such as time schedules and smart notifications, has driven smartwatches market forward. "In addition, the ongoing technological advancements are the primary factors stimulating the growth of smartwatches, as it is a completely technical product that requires constant research and development for differentiated features.", says Mordor Intelligence.

Wearable fitness electronics are also have dramatically increase in market share, however, majorities of smartwatches have already included part of their functions as a fitness tracker, which accounts for smartwatches' success as well.

Secondly, Asia is expected to be the area with highest growth rate in wearable electronic market in these years. For an example, in China wearable technologies witnessed a dramatic development, leading by Xiaomi and Huawei. Today, the number of Chinese who are willing to purchase a smartwatch or a band is increasing steadily, because the increase of citizen's income and rising awareness of healthy lifestyle.

\section{Conclusions}

In the last few decades, remarkable achievements in sensing filed, wearable electronics, novel conducting materials, etc., were generated and contributed to the high-speed development of flexible and stretchable electrodes for wearable sensors. Significant amount of wearable sensors and platforms are generated, such as the smart contact lenses, workload monitor, agricultural monitor, with which human motion monitoring, personalized healthcare, crop monitoring are stepping into a new research stage. However, many essential issues, e.g., short life time, lose accuracy during using, power sources problem, should be focused for the further development. And driven by the increasing demand for need in sports training, health monitoring and disease diagnose, enthusiasm for bio-integrated systems would maintain strong in the following years. Besides, the wearable mar- 
ket is predicted to have solid size increase with numerous needs and potential demanding not meet yet.

Interface bonding between the conducting materials and substrates that allow large deformation is vital for stretchability achieving. Stretchable structures and materials are most usual approaches for fabrication of stretchable electrodes. Among deform-able structures that include buckled structure, coiled structure, openmesh structure, etc., sponge structure seems to obtain higher stability, however the stretchability is slightly damaged. For promising materials, AgNW exhibits its extraordinary conductivity, significant harness for wearable electrodes' production. Though the Ag ion leaching and its toxicity would probably threaten people's health, many attempts, e.g., core-sheath method, were made to protect users, enabling the use of the promising materials.

Apart from developing hardware, software also faces great demanding to offer a better interaction. As huge efforts put into the building of stretchable electrodes and wearable platforms from chemistry, physics and material science, a new era with vast market and rapid flourishing of wearable electronics would be in the corner.

\section{Acknowledgements}

The author thanks Bo Wang and Erik S. YAN of University of California, Los Angeles for many stimulating discussions.

\section{Conflicts of Interest}

The author declares no conflicts of interest regarding the publication of this paper.

\section{References}

[1] Giraldo, J.P., Wu, H., Newkirk, G.M. and Kruss, S. (2019) Nanobiotechnology Approaches for Engineering Smart Plant Sensors. Nature Nanotechnology, 14, 541553. https://doi.org/10.1038/s41565-019-0470-6

[2] Zhao, S., Li, J., Cao, D., Zhang, G., Li, J., Li, K., Yang, Y., Wang, W., Jin, Y., Sun, R., et al. (2017) Recent Advancements in Flexible and Stretchable Electrodes for Electromechanical Sensors: Strategies, Materials, and Features. ACS Applied Materials \& Interfaces, 9, 12147-12164. https://doi.org/10.1021/acsami.6b13800

[3] Ray, T.R., Choi, J., Bandodkar, A.J., Krishnan, S., Gutruf, P., Tian, L., Ghaffari, R. and Rogers, J.A. (2019) Bio-Integrated Wearable Systems: A Comprehensive Review. Chemical Reviews, 119, 5461-5533. https://doi.org/10.1021/acs.chemrev.8b00573

[4] Heikenfeld, J., Jajack, A., Feldman, B., Granger, S.W., Gaitonde, S., Begtrup, G. and Katchman, B.A. (2019) Accessing Analytes in Biofluids for Peripheral Biochemical Monitoring. Nature Biotechnology, 37, 407-419. https://doi.org/10.1038/s41587-019-0040-3

[5] Seshadri, D.R., Li, R.T., Voos, J.E., Rowbottom, J.R., Alfes, C.M., Zorman, C.A. and Drummond, C.K. (2019) Wearable Sensors for Monitoring the Internal and External Workload of the Athlete. NPJ Digital Medicine, 2, 1-18. 
https://doi.org/10.1038/s41746-019-0149-2

[6] Someya, T. and Amagai, M. (2019) Toward a New Generation of Smart Skins. Nature Biotechnology, 37, 382-388. https://doi.org/10.1038/s41587-019-0079-1

[7] Wood, C.S., Thomas, M.R., Budd, J., Mashamba-Thompson, T.P., Herbst, K., Pillay, D., Peeling, R.W., Johnson, A.M., McKendry, R.A. and Stevens, M.M. (2019) Taking Connected Mobile-Health Diagnostics of Infectious Diseases to the Field. Nature, 566, 467-474. https://doi.org/10.1038/s41586-019-0956-2

[8] Kim, J., Campbell, A.S., de Ávila, B.E.-F. and Wang, J. (2019) Wearable Biosensors for Healthcare Monitoring. Nature Biotechnology, 37, 389-406. https://doi.org/10.1038/s41587-019-0045-y

[9] So, C.-F., Choi, K.-S., Wong, T.K. and Chung, J.W. (2012) Recent Advances in Noninvasive Glucose Monitoring. Medical Devices, 5, 45. https://doi.org/10.2147/MDER.S28134

[10] Núñez, C.G., Manjakkal, L. and Dahiya, R. (2019) Energy Autonomous Electronic Skin. NPJ Flexible Electronics, 3, 1-24. https://doi.org/10.1038/s41528-018-0045-x

[11] Hu, X., Li, F. and Song, Y. (2019) Wearable Power Source: A Newfangled Feasibility for Perovskite Photovoltaics. ACS Energy Letters, 4, 1065-1072. https://doi.org/10.1021/acsenergylett.9b00503

[12] Youn, D.-Y., Jung, U., Naqi, M., Choi, S.-J., Lee, M.-G., Lee, S., Park, H.-J., Kim, I.-D. and Kim, S. (2018) Wireless Real-Time Temperature Monitoring of Blood Packages: Silver Nanowire-Embedded Flexible Temperature Sensors. ACS Applied Materials \& Interfaces, 10, 44678-44685. https://doi.org/10.1021/acsami.8b11928

[13] Yang, G., Tan, W., Jin, H., Zhao, T. and Tu, L. (2019) Review Wearable Sensing System for Gait Recognition. Cluster Computing, 22, 3021-3029. https://doi.org/10.1007/s10586-018-1830-y

[14] Poitras, I., Dupuis, F., Bielmann, M., Campeau-Lecours, A., Mercier, C., Bouyer, L.J. and Roy, J.-S. (2019) Validity and Reliability of Wearable Sensors for Joint Angle Estimation: A Systematic Review. Sensors, 19, 1555. https://doi.org/10.3390/s19071555

[15] Cao, M., Xie, T. and Chen, Z. (2019) Wearable Sensors and Equipment in VR Games: A Review. In: Transactions on Edutainment $X V$, Springer, Berlin, 3-12. https://doi.org/10.1007/978-3-662-59351-6_1

[16] Pang, I., Okubo, Y., Sturnieks, D., Lord, S.R. and Brodie, M.A. (2019) Detection of near Falls Using Wearable Devices: A Systematic Review. Journal of Geriatric Physical Therapy, 42, 48-56. https://doi.org/10.1519/JPT.0000000000000181

[17] Chen, L. and Nugent, C.D. (2019) Sensorbased Activity Recognition Review. In: Human Activity Recognition and Behaviour Analysis, Springer, Berlin, 23-47. https://doi.org/10.1007/978-3-030-19408-6_2

[18] Aroganam, G., Manivannan, N. and Harrison, D. (2019) Review on Wearable technology Sensors Used in Consumer Sport Applications. Sensors, 19, 1983. https://doi.org/10.3390/s19091983

[19] Bandodkar, A.J., Jeang, W.J., Ghaffari, R. and Rogers, J.A. (2019) Wearable Sensors for Biochemical Sweat Analysis. Annual Review of Analytical Chemistry, 12, 1-22. https://doi.org/10.1146/annurev-anchem-061318-114910

[20] Seppälä, J., De Vita, I., Jämsä, T., Miettunen, J., Isohanni, M., Rubinstein, K., Feldman, Y., Grasa, E., Corripio, I., Berdun, J., et al. (2019) Mobile Phone and Wearable Sensor-Based m-Health Approaches for Psychiatric Disorders and Symptoms: Systematic Review. JMIR Mental Health, 6, e9819. https://doi.org/10.2196/mental.9819 
[21] Hechtel, N., Krückeberg, J. and Marschollek, M. (2019) Wearable Sensors for Nurses: Which Requirements Have to Be Considered? Studies in Health Technology and Informatics, 258, 241-242.

[22] Teferra, M.N., Kourbelis, C., Newman, P., Ramos, J.S., Hobbs, D., Clark, R.A. and Reynolds, K.J. (2019) Electronic Textile Electrocardiogram Monitoring in Cardiac Patients: A Scoping Review Protocol. JBI Database of Systematic Reviews and Implementation Reports, 17, 147-156. https://doi.org/10.11124/JBISRIR-2017-003630

[23] Gao, B., He, Z., He, B. and Gu, Z. (2019) Wearable Eye Health Monitoring Sensors Based on Peacock Tail-Inspired Inverse Opal Carbon. Sensors and Actuators B: Chemical, 288, 734-741. https://doi.org/10.1016/j.snb.2019.03.029

[24] Nitta, T., Terada, Z. and Hayakawa, S. (1978) Humidity Sensitive Ceramic Resistor. US Patent 4,086,556.

[25] Son, D., Lee, J., Qiao, S., Ghaffari, R., Kim, J., Lee, J.E., Song, C., Kim, S.J., Lee, D.J., Jun, S.W., et al. (2014) Multifunctional Wearable Devices for Diagnosis and Therapy of Movement Disorders. Nature Nanotechnology, 9, 397. https://doi.org/10.1038/nnano.2014.38

[26] Findlow, A., Goulermas, J., Nester, C., Howard, D. and Kenney, L. (2008) Predicting Lower Limb Joint Kinematics Using Wearable Motion Sensors. Gait \& Posture, 28, 120-126. https://doi.org/10.1016/j.gaitpost.2007.11.001

[27] Lee, S.-W., Mase, K. and Kogure, K. (2006) Detection of Spatio-Temporal Gait Parameters by Using Wearable Motion Sensors. 2005 IEEE Engineering in Medicine and Biology 27 th Annual Conference, Shanghai, 1-4 September 2005, 6836-6839.

[28] Tao, W., Liu, T., Zheng, R. and Feng, H. (2012) Gait Analysis Using Wearable Sensors. Sensors, 12, 2255-2283. https://doi.org/10.3390/s120202255

[29] Dobkin, B.H. (2013) Wearable Motion Sensors to Continuously Measure RealWorld Physical Activities. Current Opinion in Neurology, 26, 602. https://doi.org/10.1097/WCO.0000000000000026

[30] Jeon, H., Hong, S.K., Kim, M.S., Cho, S.J. and Lim, G. (2017) Omni-Purpose Stretchable Strain Sensor Based on a Highly Dense Nanocracking Structure for WholeBody Motion Monitoring. ACS Applied Materials \& Interfaces, 9, 41712-41721. https://doi.org/10.1021/acsami.7b14153

[31] Taylor, N.A., Tipton, M.J. and Kenny, G.P. (2014) Considerations for the Measurement of Core, Skin and Mean Body Temperatures. Journal of Thermal Biology, 46, 72-101. https://doi.org/10.1016/j.jtherbio.2014.10.006

[32] Chai, G., Lupan, O., Chow, L. and Heinrich, H. (2009) Crossed Zinc Oxide Nanorods for Ultraviolet Radiation Detection. Sensors and Actuators A: Physical, 150, 184-187. https://doi.org/10.1016/j.sna.2008.12.020

[33] Diffey, B.L. (2002) Sources and Measurement of Ultraviolet Radiation. Methods, 28, 4-13. https://doi.org/10.1016/S1046-2023(02)00204-9

[34] Lee, S., Jo, I., Kang, S., Jang, B., Moon, J., Park, J.B., Lee, S., Rho, S., Kim, Y. and Hong, B.H. (2017) Smart Contact Lenses with Graphene Coating for Electromagnetic Interference Shielding and Dehydration Protection. ACS Nano, 11, 5318-5324. https://doi.org/10.1021/acsnano.7b00370

[35] Kim, J., Kim, M., Lee, M.-S., Kim, K., Ji, S., Kim, Y.-T., Park, J., Na, K., Bae, K.-H., Kim, H.K., et al. (2017) Wearable Smart Sensor Systems Integrated on Soft Contact Lenses for Wireless Ocular Diagnostics. Nature Communications, 8, 14997. https://doi.org/10.1038/ncomms14997

[36] Tseng, R.C., Chen, C.-C., Hsu, S.-M. and Chuang, H.-S. (2018) Contact-Lens Bio- 
sensors. Sensors, 18, 2651. https://doi.org/10.3390/s18082651

[37] Leonardi, M., Pitchon, E.M., Bertsch, A., Renaud, P. and Mermoud, A. (2009) Wireless Contact Lens Sensor for Intraocular Pressure Monitoring: Assessment on Enucleated Pig Eyes. Acta Ophthalmologica, 87, 433-437. https://doi.org/10.1111/j.1755-3768.2008.01404.x

[38] Cortes Torres, C.C., Sampei, K., Sato, M., Raskar, R. and Miki, N. (2015) Workload Assessment with Eye Movement Monitoring Aided by Non-Invasive and Unobtrusive Micro-Fabricated Optical Sensors. Adjunct Proceedings of the 28th Annual ACM Symposium on User Interface Software \& Technology, November 2015, 53-54. https://doi.org/10.1145/2815585.2817808

[39] Yin, R., Xu, Z., Mei, M., Chen, Z., Wang, K., Liu, Y., Tang, T., Priydarshi, M.K., Meng, X., Zhao, S., et al. (2018) Soft Transparent Graphene Contact Lens Electrodes for Conformal Full-Cornea Recording of Electroretinogram. Nature Communications, 9, 2334. https://doi.org/10.1038/s41467-018-04781-w

[40] Park, J., Kim, J., Kim, S.-Y., Cheong, W.H., Jang, J., Park, Y.-G., Na, K., Kim, Y.-T., Heo, J.H., Lee, C.Y., et al. (2018) Soft, Smart Contact Lenses with Integrations of Wireless Circuits, Glucose Sensors, and Displays. Science Advances, 4, eaap9841. https://doi.org/10.1126/sciadv.aap9841

[41] Oliver, N., Toumazou, C., Cass, A. and Johnston, D. (2009) Glucose Sensors: A Review of Current and Emerging Technology. Diabetic Medicine, 26, 197-210. https://doi.org/10.1111/j.1464-5491.2008.02642.x

[42] Chen, G.-Z., Chan, I.-S. and Lam, D.C. (2013) Capacitive Contact Lens Sensor for Continuous Non-Invasive Intraocular Pressure Monitoring. Sensors and Actuators A: Physical, 203, 112-118. https://doi.org/10.1016/j.sna.2013.08.029

[43] Besteman, K., Lee, J.-O., Wiertz, F.G., Heering, H.A. and Dekker, C. (2003) Enzyme-Coated Carbon Nanotubes as Single Molecule Biosensors. Nano Letters, 3, 727730. https://doi.org/10.1021/nl034139u

[44] Chen, R.J., Zhang, Y., Wang, D. and Dai, H. (2001) Noncovalent Sidewall Functionalization of Single-Walled Carbon Nanotubes for Protein Immobilization. Journal of the American Chemical Society, 123, 3838-3839.

https://doi.org/10.1021/ja010172b

[45] Walker, E.J., McAinch, A.J., Sweeting, A. and Aughey, R.J. (2016) Inertial Sensors to Estimate the Energy Expenditure of Team-Sport Athletes. Journal of Science and Medicine in Sport, 19, 177-181. https://doi.org/10.1016/j.jsams.2015.01.013

[46] Shcherbina, A., Mattsson, C.M., Waggott, D., Salisbury, H., Christle, J.W., Hastie, T., Wheeler, M.T. and Ashley, E.A. (2017) Accuracy in Wrist-Worn, Sensor-Based Measurements of Heart Rate and Energy Expenditure in a Diverse Cohort. Journal of Personalized Medicine, 7, 3. https://doi.org/10.3390/jpm7020003

[47] Wang, S., Xu, J., Wang, W., Wang, G.-J.N., Rastak, R., Molina-Lopez, F., Chung, J.W., Niu, S., Feig, V.R., Lopez, J., et al. (2018) Skin Electronics from Scalable Fabrication of an Intrinsically Stretchable Transistor Array. Nature, 555, 83. https://doi.org/10.1038/nature25494

[48] Lipscomb, C.E. (2000) Medical Subject Headings (Mesh). Bulletin of the Medical Library Association, 88, 265.

[49] Pandian, P., Mohanavelu, K., Safeer, K., Kotresh, T., Shakunthala, D., Gopal, P. and Padaki, V. (2008) Smart Vest: Wearable Multi-Parameter Remote Physiological Monitoring System. Medical Engineering \& Physics, 30, 466-477. https://doi.org/10.1016/j.medengphy.2007.05.014

[50] Wang, Y., Wang, L., Yang, T., Li, X., Zang, X., Zhu, M., Wang, K., Wu, D. and Zhu, 
H. (2014) Wearable and Highly Sensitive Graphene Strain Sensors for Human Motion Monitoring. Advanced Functional Materials, 24, 4666-4670. https://doi.org/10.1002/adfm.201400379

[51] da Silva, A.F., Gonçalves, A.F., de Almeida Ferreira, L.A., Araújo, F.M.M., Mendes, P.M. and Correia, J.H. (2010) A Smart Skin PVC Foil Based on FBG Sensors for Monitoring Strain and Temperature. IEEE Transactions on Industrial Electronics, 58, 2728-2735. https://doi.org/10.1109/TIE.2010.2057233

[52] Gao, W., Emaminejad, S., Nyein, H.Y.Y., Challa, S., Chen, K., Peck, A., Fahad, H.M., Ota, H., Shiraki, H., Kiriya, D., et al. (2016) Fully Integrated Wearable Sensor Arrays for Multiplexed in Situ Perspiration Analysis. Nature, 529, 509. https://doi.org/10.1038/nature16521

[53] Lima, A. and Bakker, J. (2006) Noninvasive Monitoring of Peripheral Perfusion. In: Applied Physiology in Intensive Care Medicine, Springer, Berlin, 131-141. https://doi.org/10.1007/3-540-37363-2_26

[54] Demirer, G.S., Zhang, H., Matos, J.L., Goh, N.S., Cunningham, F.J., Sung, Y., Chang, R., Aditham, A.J., Chio, L., Cho, M.-J., et al. (2019) High Aspect Ratio Nanomaterials Enable Delivery of Functional Genetic Material without DNA Integration in Mature Plants. Nature Nanotechnology, 14, 456. https://doi.org/10.1038/s41565-019-0382-5

[55] Ibrahim, E.R., Hossain, M., Roslan, H.A., et al. (2014) Genetic Transformation of Metroxylon sagu (Rottb.) Cultures via Agrobacterium-Mediated and Particle Bombardment. BioMed Research International, 2014, Article ID: 348140. https://doi.org/10.1155/2014/348140

[56] Chilton, M.-D., Drummond, M.H., Merlo, D.J., Sciaky, D., Montoya, A.L., Gordon, M.P. and Nester, E.W. (1977) Stable Incorporation of Plasmid DNA into Higher Plant Cells: The Molecular Basis of Crown Gall Tumorigenesis. Cell, 11, 263-271. https://doi.org/10.1016/0092-8674(77)90043-5

[57] Gelvin, S.B. (2003) Agrobacterium Mediated Plant Transformation: The Biology behind the "Gene-Jockeying" Tool. Microbiology and Molecular Biology Reviews, 67, 16-37. https://doi.org/10.1128/MMBR.67.1.16-37.2003

[58] Kwak, S.-Y., Lew, T.T.S., Sweeney, C.J., Koman, V.B., Wong, M.H., Bohmert Tatarev, K., Snell, K.D., Seo, J.S., Chua, N.-H. and Strano, M.S. (2019) Chloroplastselective Gene Delivery and Expression in Planta Using Chitosan-Complexed Single Walled Carbon Nanotube Carriers. Nature Nanotechnology, 14, 447.

https://doi.org/10.1038/s41565-019-0375-4

[59] Ortega-Villasante, C., Burén, S., BarónSola, Á., Martínez, F. and Hernández, L.E. (2016) In Vivo ROS and Redox Potential Fluorescent Detection in Plants: Present Approaches and Future Perspectives. Methods, 109, 92-104.

https://doi.org/10.1016/j.ymeth.2016.07.009

[60] Kanchiswamy, C., Malnoy, M., Occhipinti, A. and Maffei, M. (2014) Calcium Imaging Perspectives in Plants. International Journal of Molecular Sciences, 15, 3842-3859. https://doi.org/10.3390/ijms15033842

[61] Köhler, B. and Blatt, M.R. (2002) Protein Phosphorylation Activates the Guard Cell $\mathrm{Ca}^{2+}$ Channel and Is a Prerequisite for Gating by Abscisic Acid. The Plant Journal, 32, 185-194. https://doi.org/10.1046/j.1365-313X.2002.01414.x

[62] Garcia-Mata, C., Gay, R., Sokolovski, S., Hills, A., Lamattina, L. and Blatt, M.R. (2003) Nitric Oxide Regulates K+ and Clchannels in Guard Cells through a Subset of Abscisic Acid-Evoked Signaling Pathways. Proceedings of the National Academy of Sciences, 100, 11116-11121. https://doi.org/10.1073/pnas.1434381100

[63] Zhu, Q., Wang, L., Dong, Q., Chang, S., Wen, K., Jia, S., Chu, Z., Wang, H., Gao, P., 
Zhao, H., et al. (2017) Fret-Based Glucose Imaging Identifies Glucose Signalling in Response to Biotic and Abiotic Stresses in Rice Roots. Journal of Plant Physiology, 215, 6572. https://doi.org/10.1016/j.jplph.2017.05.007

[64] Chaudhuri, B., Hörmann, F., Lalonde, S., Brady, S.M., Orlando, D.A., Benfey, P. and Frommer, W.B. (2008) Protonophore- and Ph-Insensitive Glucose and Sucrose Accumulation Detected by Fret Nanosensors in Arabidopsis Root Tips. The Plant Journal, 56, 948-962. https://doi.org/10.1111/j.1365-313X.2008.03652.x

[65] Mur, L.A., Mandon, J., Cristescu, S.M., Harren, F.J. and Prats, E. (2011) Methods of Nitric Oxide Detection in Plants: A Commentary. Plant Science, 181, 509-519. https://doi.org/10.1016/j.plantsci.2011.04.003

[66] López-Carbonell, M., Gabasa, M. and Jáuregui, O. (2009) Enhanced Determination of Abscisic Acid (ABA) and Abscisic Acid Glucose Ester (ABA-GE) in Cistus albidus Plants by Liquid Chromatographymass Spectrometry in Tandem Mode. Plant Physiology and Biochemistry, 47, 256-261. https://doi.org/10.1016/j.plaphy.2008.12.016

[67] Xie, S., Wang, F. and Chen, Z. (2013) Determination of Endogenous Jasmonic Acid in Plant Samples by Liquid Chromatography Electrochemical Detection Based on Derivatization with Dopamine. Analyst, 138, 1226-1231.

https://doi.org/10.1039/c2an36455g

[68] Engelberth, J., Schmelz, E.A., Alborn, H.T., Cardoza, Y.J., Huang, J. and Tumlinson, J.H. (2003) Simultaneous Quantification of Jasmonic Acid and Salicylic Acid in Plants by Vapor-Phase Extraction and Gas Chromatography-Chemical Ionizationmass Spectrometry. Analytical Biochemistry, 312, 242-250. https://doi.org/10.1016/S0003-2697(02)00466-9

[69] Seskar, M., Shulaev, V. and Raskin, I. (1998) Endogenous Methyl Salicylate in Pathogen-Inoculated Tobacco Plants. Plant Physiology, 116, 387-392.

https://doi.org/10.1104/pp.116.1.387

[70] Pantea, D., Darmstadt, H., Kaliaguine, S. and Roy, C. (2003) Electrical Conductivity of Conductive Carbon Blacks: Influence of Surface Chemistry and Topology. Applied Surface Science, 217, 181-193. https://doi.org/10.1016/S0169-4332(03)00550-6

[71] Wu, X., Han, Y., Zhang, X., Zhou, Z. and Lu, C. (2016) Large-Area Compliant, Low-Cost, and Versatile Pressure-Sensing Platform Based on Microcrack-Designed Carbon Black@ Polyurethane Sponge for Human Machine Interfacing. Advanced Functional Materials, 26, 6246-6256. https://doi.org/10.1002/adfm.201601995

[72] Yu, M.-F., Lourie, O., Dyer, M.J., Moloni, K., Kelly, T.F. and Ruoff, R.S. (2000) Strength and Breaking Mechanism of Multiwalled Carbon Nanotubes under Tensile Load. Science, 287, 637-640. https://doi.org/10.1126/science.287.5453.637

[73] Hong, S. and Myung, S. (2007) Nanotube Electronics: A Flexible Approach to Mobility. Nature Nanotechnology, 2, 207. https://doi.org/10.1038/nnano.2007.89

[74] Li, Z., Ma, G., Ge, R., Qin, F., Dong, X., Meng, W., Liu, T., Tong, J., Jiang, F., Zhou, Y., et al. (2016) Free-Standing Conducting Polymer Films for High-Performance Energy Devices. Angewandte Chemie International Edition, 55, 979-982. https://doi.org/10.1002/anie.201509033

[75] Wei, Y., Chen, S., Yuan, X., Wang, P. and Liu, L. (2016) Multiscale Wrinkled Microstructures for Piezoresistive Fibers. Advanced Functional Materials, 26, 50785085. https://doi.org/10.1002/adfm.201600580

[76] Akter, T. and Kim, W.S. (2012) Reversibly Stretchable Transparent Conductive Coatings of Spray-Deposited Silver Nanowires. ACS Applied Materials \& Interfaces, 4, 1855-1859. https://doi.org/10.1021/am300058j 
[77] Hu, W., Wang, R., Lu, Y. and Pei, Q. (2014) An Elastomeric Transparent Composite Electrode Based on Copper Nanowires and Polyurethane. Journal of Materials Chemistry C, 2, 1298-1305. https://doi.org/10.1039/C3TC31647E

[78] Jung, S.M., Preston, D.J., Jung, H.Y., Deng, Z., Wang, E.N. and Kong, J. (2016) Porous CU Nanowire Aerosponges from One Step Assembly and Their Applications in Heat Dissipation. Advanced Materials, 28, 1413-1419. https://doi.org/10.1002/adma.201504774

[79] Zhang, Y., Zhu, P., Li, G., Zhao, T., Fu, X., Sun, R., Zhou, F. and Wong, C.-P. (2013) Facile Preparation of Monodisperse, Impurity-Free, and Antioxidation Copper Nanoparticles on a Large Scale for Application in Conductive Ink. ACS Applied Materials \& Interfaces, 6, 560-567. https://doi.org/10.1021/am404620y

[80] Radha, B., Sagade, A.A. and Kulkarni, G. (2011) Flexible and Semitransparent Strain Sensors Based on Micromolded PD Nanoparticle-Carbon $\mu$-Stripes. ACS Applied Materials \& Interfaces, 3, 2173-2178. https://doi.org/10.1021/am2002873

[81] Hu, Y., Zhao, T., Zhu, P., Zhu, Y., Shuai, X., Liang, X., Sun, R., Lu, D.D. and Wong, C.-P. (2016) Low Cost and Highly Conductive Elastic Composites for Flexible and Printable Electronics. Journal of Materials Chemistry C, 4, 5839-5848. https://doi.org/10.1039/C6TC01340F

[82] Zhou, A., Sim, R., Luo, Y. and Gao, X. (2017) High-Performance Stretchable Electrodes Prepared from Elastomeric Current Collectors and Binders. Journal of Materials Chemistry A, 5, 21550-21559. https://doi.org/10.1039/C7TA07185J

[83] Amjadi, M., Pichitpajongkit, A., Lee, S., Ryu, S. and Park, I. (2014) Highly Stretchable and Sensitive Strain Sensor Based on Silver Nanowire-Elastomer Nanocomposite. ACS Nano, 8, 5154-5163. https://doi.org/10.1021/nn501204t

[84] Liu, Z., Fang, S., Moura, F.A., Ding, J., Jiang, N., Di, J., Zhang, M., Lepró, X., Galvao, D., Haines, C., et al. (2015) Hierarchically Buckled Sheath-Core Fibers for Superelastic Electronics, Sensors, and Muscles. Science, 349, 400-404. https://doi.org/10.1126/science.aaa7952

[85] Yang, T., Wang, W., Zhang, H., Li, X., Shi, J., He, Y., Zheng, Q.-S., Li, Z. and Zhu, H. (2015) Tactile Sensing System Based on Arrays of Graphene Woven Microfabrics: Electromechanical Behavior and Electronic Skin Application. ACS Nano, 9, 1086710875. https://doi.org/10.1021/acsnano.5b03851

[86] Shang, Y., He, X., Li, Y., Zhang, L., Li, Z., Ji, C., Shi, E., Li, P., Zhu, K., Peng, Q., et al. (2012) Super-Stretchable Spring-Like Carbon Nanotube Ropes. Advanced Materials, 24, 2896-2900. https://doi.org/10.1002/adma.201200576

[87] Ge, J., Yao, H.-B., Wang, X., Ye, Y.-D., Wang, J.-L., Wu, Z.-Y., Liu, J.-W., Fan, F.-J., Gao, H.L., Zhang, C.-L., et al. (2013) Stretchable Conductors Based on Silver Nanowires: Improved Performance through a Binary Network Design. Angewandte Chemie International Edition, 52, 1654-1659. https://doi.org/10.1002/anie.201209596

[88] Lee, J., Kwon, H., Seo, J., Shin, S., Koo, J.H., Pang, C., Son, S., Kim, J.H., Jang, Y.H., Kim, D.E., et al. (2015) Conductive Fiber-Based Ultrasensitive Textile Pressure Sensor for Wearable Electronics. Advanced Materials, 27, 2433-2439. https://doi.org/10.1002/adma.201500009

[89] Cheng, Y., Wang, R., Sun, J. and Gao, L. (2015) A Stretchable and Highly Sensitive Graphene-Based Fiber for Sensing Tensile Strain, Bending, and Torsion. Advanced Materials, 27, 7365-7371. https://doi.org/10.1002/adma.201503558

[90] Han, S., Hong, S., Ham, J., Yeo, J., Lee, J., Kang, B., Lee, P., Kwon, J., Lee, S.S., Yang, M.-Y., et al. (2014) Fast Plasmonic Laser Nanowelding for a Cu-Nanowire Percolation Network for Flexible Transparent Conductors and Stretchable Electronics. $A d$ - 
vanced Materials, 26, 5808-5814. https://doi.org/10.1002/adma.201400474

[91] Lu, N., Lu, C., Yang, S. and Rogers, J. (2012) Highly Sensitive Skin-Mountable Strain Gauges Based Entirely on Elastomers. Advanced Functional Materials, 22, 40444050. https://doi.org/10.1002/adfm.201200498

[92] Gutruf, P., Walia, S., Ali Nur, M., Sriram, S. and Bhaskaran, M. (2014) Strain Response of Stretchable Micro-Electrodes: Controlling Sensitivity with Serpentine Designs and Encapsulation. Applied Physics Letters, 104, Article ID: 021908. https://doi.org/10.1063/1.4862264

[93] Dinh, T., Phan, H.-P., Nguyen, T.-K., Qamar, A., Foisal, A.R.M., Viet, T.N., Tran, C.D., Zhu, Y., Nguyen, N.-T. and Dao, D.V. (2016) Environment-Friendly Carbon Nanotube Based Flexible Electronics for Noninvasive and Wearable Healthcare. Journal of Materials Chemistry C, 4, 10061-10068. https://doi.org/10.1039/C6TC02708C

[94] Wang, S., Huang, Y. and Rogers, J.A. (2015) Mechanical Designs for Inorganic Stretchable Circuits in Soft Electronics. IEEE Transactions on Components, Packaging and Manufacturing Technology, 5, 1201-1218. https://doi.org/10.1109/TCPMT.2015.2417801

[95] Trung, T.Q. and Lee, N.-E. (2017) Recent Progress on Stretchable Electronic Devices with Intrinsically Stretchable Components. Advanced Materials, 29. https://doi.org/10.1002/adma.201603167

[96] Jeong, C.K., Lee, J., Han, S., Ryu, J., Hwang, G.T., Park, D.Y., Park, J.H., Lee, S.S., Byun, M., Ko, S.H., et al. (2015) A Hyper-Stretchable Elastic-Composite Energy Harvester. Advanced Materials, 27, 2866-2875. https://doi.org/10.1002/adma.201500367

[97] Dankoco, M., Tesfay, G., Bènevent, E. and Bendahan, M. (2016) Temperature Sensor Realized by Inkjet Printing Process on Flexible Substrate. Materials Science and Engineering. B, 205, 1-5. https://doi.org/10.1016/j.mseb.2015.11.003

[98] Hu, L., Kim, H.S., Lee, J.-Y., Peumans, P. and Cui, Y. (2010) Scalable Coating and Properties of Transparent, Flexible, Silver Nanowire Electrodes. ACS Nano, 4, 29552963. https://doi.org/10.1021/nn1005232

[99] Chen, Y., Lu, B., Chen, Y. and Feng, X. (2015) Breathable and Stretchable Temperature Sensors Inspired by Skin. Scientific Reports, 5, Article No. 11505. https://doi.org/10.1038/srep11505

[100] Liu, C.-H. and Yu, X. (2011) Silver Nanowire-Based Transparent, Flexible, and Conductive Thin Film. Nanoscale Research Letters, 6, 75. https://doi.org/10.1186/1556-276X-6-75

[101] Bobinger, M., Angeli, D., Colasanti, S., La Torraca, P., Larcher, L. and Lugli, P. (2017) Infrared, Transient Thermal, and Electrical Properties of Silver Nanowire Thin Films for Transparent Heaters and Energy Efficient Coatings. Physica Status Solidi (A), 214, Article ID: 1600466. https://doi.org/10.1002/pssa.201600466

[102] Xu, F. and Zhu, Y. (2012) Highly Conductive and Stretchable Silver Nanowire Conductors. Advanced Materials, 24, 5117-5122. https://doi.org/10.1002/adma.201201886

[103] Lee, D., Youn, D.-Y., Luo, Z. and Kim, I.-D. (2016) Highly Flexible Transparent Electrodes Using a Silver Nanowires-Embedded Colorless Polyimide Film via Chemical Modification. RSC Advances, 6, 30331-30336.

https://doi.org/10.1039/C6RA03200A

[104] Kim, Y., Ryu, T.I., Ok, K.-H., Kwak, M.-G., Park, S., Park, N.-G., Han, C.J., Kim, B.S., Ko, M.J., Son, H.J., et al. (2015) Inverted Layer-by-Layer Fabrication of an Ul- 
traflexible and Transparent AG Nanowire/Conductive Polymer Composite Electrode for Use in High-Performance Organic Solar Cells. Advanced Functional Materials, 25, 4580-4589. https://doi.org/10.1002/adfm.201570199

[105] Nam, S., Song, M., Kim, D.-H., Cho, B., Lee, H.M., Kwon, J.-D., Park, S.-G., Nam, K.S., Jeong, Y., Kwon, S.-H., et al. (2014) Ultrasmooth, Extremely Deformable and Shape Recoverable AG Nanowire Embedded Transparent Electrode. Scientific Reports, 4, Article No. 4788. https://doi.org/10.1038/srep04788

[106] Im, H.-G., Jung, S.-H., Jin, J., Lee, D., Lee, J., Lee, D., Lee, J.-Y., Kim, I.-D. and Bae, B.-S. (2014) Flexible Transparent Conducting Hybrid Film Using a Surfaceembedded Copper Nanowire Network: A Highly Oxidation-Resistant Copper Nanowire Electrode for Flexible Optoelectronics. ACS Nano, 8, 10973-10979. https://doi.org/10.1021/nn504883m

[107] McShan, D., Ray, P.C. and Yu, H. (2014) Molecular Toxicity Mechanism of Nanosilver. Journal of Food and Drug Analysis, 22, 116-127. https://doi.org/10.1016/j.jfda.2014.01.010

[108] Choi, S., Han, S.I., Jung, D., Hwang, H.J., Lim, C., Bae, S., Park, O.K., Tschabrunn, C.M., Lee, M., Bae, S.Y., et al. (2018) Highly Conductive, Stretchable and Biocompatible AG-AU Core-Sheath Nanowire Composite for Wearable and Implantable Bioelectronics. Nature Nanotechnology, 13, 1048-1056. https://doi.org/10.1038/s41565-018-0226-8

[109] Liang, J., Li, L., Niu, X., Yu, Z. and Pei, Q. (2013) Elastomeric Polymer Light-Emitting Devices and Displays. Nature Photonics, 7, 817. https://doi.org/10.1038/nphoton.2013.242

[110] Li, X., Gao, H., Murphy, C.J. and Caswell, K. (2003) Nanoindentation of Silver Nanowires. Nano Letters, 3, 1495-1498. https://doi.org/10.1021/nl034525b

[111] Caswell, K., Bender, C.M. and Murphy, C.J. (2003) Seedless, Surfactantless Wet Chemical Synthesis of Silver Nanowires. Nano Letters, 3, 667-669. https://doi.org/10.1021/nl0341178

[112] Sun, Y., Yin, Y., Mayers, B.T., Herricks, T. and Xia, Y. (2002) Uniform Silver Nanowires Synthesis by Reducing agno3 with Ethylene Glycol in the Presence of Seeds and Poly(vinyl pyrrolidone) Chemistry of Materials, 14, 4736-4745.

https://doi.org/10.1021/cm020587b

[113] Liu, S., Yue, J. and Gedanken, A. (2001) Synthesis of Long Silver Nanowires from AGBR Nanocrystals. Advanced Materials, 13, 656-658. https://doi.org/10.1002/1521-4095(200105)13:9<656::AID-ADMA656>3.0.CO;2-O

[114] Wang, S., Tian, Y., Hang, C. and Wang, C. (2018) Cohesively Enhanced Electrical Conductivity and Thermal Stability of Silver Nanowire Networks by Nickel Ion Bridge Joining. Scientific Reports, 8, Article No. 5260. https://doi.org/10.1038/s41598-018-21777-0

[115] Yun, Y.S., Kim, D.H., Kim, B., Park, H.H. and Jin, H.-J. (2012) Transparent Conducting Films Based on Graphene Oxide/Silver Nanowire Hybrids with High Flexibility. Synthetic Metals, 162, 1364-1368. https://doi.org/10.1016/j.synthmet.2012.05.026

[116] Peng, Y. and Chen, Q. (2011) Simplified Fabrication and Electrical Analysis of Crystalline Silver Nanowire. Applied Physics A, 105, 841-846. https://doi.org/10.1007/s00339-011-6635-2

[117] Dohn, S., Mølhave, K. and Bøggild, P. (2005) Direct Measurement of Resistance of Multiwalled Carbon Nanotubes Using Micro Four-Point Probes. Sensor Letters, 3, 300-303. https://doi.org/10.1166/sl.2005.041

[118] Tang, B. and Ngan, A. (2004) Investigation of Viscoelastic Properties of Amorphous 
Selenium near Glass Transition Using Depth Sensing Indentation. Soft Materials, 2, 125-144. https://doi.org/10.1081/SMTS-200056116

[119] Pharr, G. (1998) Measurement of Mechanical Properties by Ultra-Low Load Indentation. Materials Science and Engineering: A, 253, 151-159. https://doi.org/10.1016/S0921-5093(98)00724-2

[120] Christopher, D., Smith, R. and Richter, A. (2001) Atomistic Modelling of Nanoindentation in Iron and Silver. Nanotechnology, 12, 372. https://doi.org/10.1088/0957-4484/12/3/328

[121] Ma, Q. and Clarke, D.R. (1995) Size Dependent Hardness of Silver Single Crystals. Journal of Materials Research, 10, 853-863. https://doi.org/10.1557/JMR.1995.0853

[122] Zhao, M., Slaughter, W.S., Li, M. and Mao, S.X. (2003) Material-Length Scale Controlled Nanoindentation Size Effects Due to Strain-Gradient Plasticity. Acta Materialia, 51, 4461-4469. https://doi.org/10.1016/S1359-6454(03)00281-7

[123] Kim, M.J. and Shin, S. (2014) Toxic Effects of Silver Nanoparticles and Nanowires on Erythrocyte Rheology. Food and Chemical Toxicology, 67, 80-86.

https://doi.org/10.1016/j.fct.2014.02.006

[124] Gong, S., Schwalb, W., Wang, Y., Chen, Y., Tang, Y., Si, J., Shirinzadeh, B. and Cheng, W. (2014) A Wearable and Highly Sensitive Pressure Sensor with Ultrathin Gold Nanowires. Nature Communications, 5, 3132. https://doi.org/10.1038/ncomms4132

[125] Chen, Y., Ouyang, Z., Gu, M. and Cheng, W. (2013) Mechanically Strong, Optically Transparent, Giant Metal Superlattice Nanomembranes from Ultrathin Gold Nanowires. Advanced Materials, 25, 80-85. https://doi.org/10.1002/adma.201202241

[126] Hou, T.-C., Yang, Y., Zhang, H., Chen, J., Chen, L.-J. and Wang, Z.L. (2013) Triboelectric Nanogenerator Built inside Shoe Insole for Harvesting Walking Energy. Nano Energy, 2, 856-862. https://doi.org/10.1016/j.nanoen.2013.03.001 


\section{Nomenclature}

The abbreviations used in this paper are shown below:

Table A. Explanation of Abbreviations in this paper.

\begin{tabular}{llll}
\hline Abbreviation & Definition & Abbreviation & Definition \\
\hline AgNW & Silver nanowires & GSI & Gadd severity index \\
CNT & Carbon nanotube & HITsp & $\begin{array}{l}\text { Head impact telemetry } \\
\text { severity profile }\end{array}$ \\
CB & Carbon black & IMU & Inertial or magnetic units \\
GO & Graphene oxide & PPG & Photoplethysmography \\
PPy & Polypyrrole & VCG & Vectorcardiogram \\
NP & Nanoparticle & SCD & Sudden cardiac death \\
AR & Augmented reality & EOG & Electrooculogram \\
VR & Visual reality & ECG & Electrocardiography \\
HR & Heart Rate & EEG & Electroencephalography \\
IOC & Inverse opal carbon & EMG & Electromyography \\
DR & Diabetic retinopathy & GWF & Electromyography \\
DES & Dry eye syndrome & SWCNT & Singlewalled carbon \\
nanotubes
\end{tabular}

